NBER WORKING PAPER SERIES

\title{
SHRINKAGE ESTIMATION OF HIGH-DIMENSIONAL FACTOR MODELS WITH STRUCTURAL INSTABILITIES
}

\author{
Xu Cheng \\ Zhipeng Liao \\ Frank Schorfheide \\ Working Paper 19792 \\ http://www.nber.org/papers/w19792 \\ NATIONAL BUREAU OF ECONOMIC RESEARCH \\ 1050 Massachusetts Avenue \\ Cambridge, MA 02138 \\ January 2014
}

Minchul Shin (Penn) provided excellent research assistance. Many thanks to Ataman Ozylidirim for granting us with access to a selected set of time series published by The Conference Board. We also thank Xu Han and seminar participants at the University of Pennsylvania, Yale University, the 2013 Montreal Time Series Conference, the 2013 Tsinghua Econometrics Conference, the 2013 NSF-NBER Time Series Conference, and the $2013 \mathrm{New}$ York Area Econometrics Colloquium for helpful comments and suggestions. Schorfheide gratefully acknowledges financial support from the National Science Foundation under Grant SES 1061725. The views expressed herein are those of the authors and do not necessarily reflect the views of the National Bureau of Economic Research.

NBER working papers are circulated for discussion and comment purposes. They have not been peerreviewed or been subject to the review by the NBER Board of Directors that accompanies official NBER publications.

(C) 2014 by Xu Cheng, Zhipeng Liao, and Frank Schorfheide. All rights reserved. Short sections of text, not to exceed two paragraphs, may be quoted without explicit permission provided that full credit, including $\odot$ notice, is given to the source. 
Shrinkage Estimation of High-Dimensional Factor Models with Structural Instabilities

$\mathrm{Xu}$ Cheng, Zhipeng Liao, and Frank Schorfheide

NBER Working Paper No. 19792

January 2014

JEL No. C13,C33,C52

\begin{abstract}
$\underline{\text { ABSTRACT }}$
In high-dimensional factor models, both the factor loadings and the number of factors may change over time. This paper proposes a shrinkage estimator that detects and disentangles these instabilities. The new method simultaneously and consistently estimates the number of pre- and post-break factors, which liberates researchers from sequential testing and achieves uniform control of the family-wise model selection errors over an increasing number of variables. The shrinkage estimator only requires the calculation of principal components and the solution of a convex optimization problem, which makes its computation efficient and accurate. The finite sample performance of the new method is investigated in Monte Carlo simulations. In an empirical application, we study the change in factor loadings and emergence of new factors during the Great Recession.
\end{abstract}

\section{Xu Cheng}

Department of Economics

University of Pennsylvania

3718 Locust Walk

Philadelphia, PA 19104

xucheng@econ.upenn.edu

Zhipeng Liao

Department of Economics

University of California, Los Angeles

Bunche Hall

Los Angeles, CA 90095

zhipeng.liao@econ.ucla.edu
Frank Schorfheide

University of Pennsylvania

Department of Economics

3718 Locust Walk

Philadelphia, PA 19104-6297

and NBER

schorf@ssc.upenn.edu

An online appendix is available at:

http://www.nber.org/data-appendix/w19792 


\section{Introduction}

High-dimensional factor models are widely used to analyze macroeconomic and financial panel data, where a small number of unobserved factors drive the comovement of a large number of time series. This paper focuses on the complications in the estimation of factor models that arise from potential structural breaks. Our leading empirical example of a potential structural break is the beginning of the 2007-2009 (Great) recession, which, unlike other post-war U.S. recessions, was characterized by a severe disruption of financial markets, a slow recovery, and a lasting episode of zero nominal interest rates and unconventional monetary policies. Throughout this paper, we distinguish between two types of factor model instabilities: changes in the factor loadings, which alter the response of macroeconomic variables to a fixed number of underlying factors (type-1 instability), and changes in the number of factors (type-2 instability). Since only the product of factors and loadings is identifiable, we use a normalization that attributes changes in this product to changes in loadings.

The main contribution of this paper is in providing a novel econometric procedure that allows researchers to consistently estimate the number of pre- and post-break factors simultaneously and to consistently detect changes in factor loadings in cases in which the number of factors remaines unchanged. Our estimation method relies on a penalized least squares (PLS) criterion function in which adaptive group-LASSO penalties are attached to pre-break factor loadings, to coefficients that parameterize changes in factor loadings after the break, and to coefficients that are associated with new post-break factors. The PLS estimator is a shrinkage estimator because, as compared with the unrestricted least squares estimator, it sets small coefficient estimates equal to zero. The number of factors is determined based on the number of nonzero columns in the loading matrices, and we refer to the determination of the number of factors and the presence of breaks as model selection.

Our procedure substantially differs from existing methods in several dimensions. First, to detect instabilities in factor loadings, our method does not require knowledge of the factors before and/or after the break. Second, our procedure automatically determines whether a structural break belongs to the type- 1 or type- 2 category. Third, for type- 2 instabilities, we decompose the structural change into the contribution of new factors and the contribution of changes of loadings for the old factors.

Fourth, the consistency of our procedure is preserved even if the break date is unknown. 
It is known in the literature (e.g., Breitung and Eickmeier (2011)) that the presence of instabilities leads to the overestimation of the number of factors. In turn, a misspecification of a break date leads to the overestimation of pre- or post-break factors. Thus, roughly speaking, we search for break dates that minimize the sum of the number of pre- and postbreak factors. While this procedure itself does not deliver a consistent estimate of the break date, it does generate consistent estimates of the number of pre- and post-break factors even if the "true" break date is unknown to the researcher.

The empirical analysis in this paper revisits a recent study by Stock and Watson (2012), who investigated whether new factors appeared at the onset of the Great Recession, considering a large data set of macroeconomic and financial times series. In a nutshell, Stock and Watson (2012) extended the pre-break factor to the post-break period and examined whether there was evidence of an un-modeled factor in the residuals of the post-break sample. They found no such evidence. Our empirical results differ. Using a similar set of time series, but sampled at a monthly frequency, we find evidence of a type- 2 instability at the beginning of the Great Recession (i.e., the emergence of a new factor, which can be interpreted as a financial factor). Our estimation results also indicate that the factor loadings changed drastically during this episode. Because Stock and Watson (2012) normalized the size of the loadings rather than the variance of the factors in their analysis, the change in loadings in our analysis mirrors the increase in factor volatility in their analysis.

We are building on a large body of literature on the analysis of factor models. In a seminal paper, Bai and $\mathrm{Ng}$ (2002) provide information criteria to select the number of factors in stable factor models. We use their assumptions about cross-sectional and temporal dependence and heteroskedasticity in the idiosyncratic errors as a starting point for our theoretical analysis. In subsequent work, several authors (e.g., Onatski (2010), Alessi, Barigozzi, and Capasso (2010), Kapetanios (2010), Caner and Han (2012), Ahn and Horenstein (2013), and Choi (2013)) proposed alternative methods for estimating the number of factors in a stable environment.

Stock and Watson (2002) and, more recently, Bates, Plagborg-Møller, Stock, and Watson (2013), show that in the presence of small structural instabilities of the factor loadings the principal component estimator of the factors remains consistent. Our paper focuses on large structural breaks that render the principal component estimator inconsistent. As shown, for instance, in Breitung and Eickmeier (2011), a factor model with big structural breaks can always be written as a stable model with a larger number of pseudo-true factors. These 
pseudo-true factors are comparable to the factors that are being estimated with a static factor model, if the "true" factors are current and lagged values of a lower-dimensional vector of dynamic factors (e.g., Forni, Hallin, Lippi, and Reichlin (2000), Amengual and Watson (2007), Bai and Ng (2007), Hallin and Lika (2007), Onatski (2009), and Breitung and Pigorsch (2013)). The above-mentioned methods for estimating the number of factors in a stable environment, are only able to determine the number of pseudo-true factors, but not the actual number of pre- and post-break factors. However, in many applications (e.g., forecasting with factor-augmented autoregressive models) the consistent estimation of the post-break factors is crucial.

Several structural break tests for factor models have been developed in the literature. Most papers, including Stock and Watson (2009), Breitung and Eickmeier (2011), Chen, Dolado, and Gonzalo (2011), Han and Inoue (2011), and Corradi and Swanson (2013), consider the null hypothesis of no change in loadings versus an alternative of a single break in the loadings, assuming that the number of factors stays constant throughout the sample. The key challenges are to ensure that the estimation error with the factors is asymptotically negligible and to control the family-wise error as the number of factor loadings, and hence the number of parameters on which the null hypothesis is imposed, tends to infinity in the large sample approximations. Our PLS estimation approach automatically controls the familywise error over an increasing number of variables. Furthermore, to achieve consistency, we only require that the number of the time series variables and the number of time periods are both large without any restriction on their relative rates, whereas structural break tests in the literature typically restrict their relative rates to ensure that the generated-regressor effect is negligible.

If the break point is known one, could study the emergence of new post-break factors simply by applying one of the existing methods for determining the number of factors in a stable environment to the pre- and post-break subsamples. Alternatively, Stock and Watson (2012) test for the presence of a factor in the errors associated with the forecasts of the postbreak observations based on extensions of the pre-break factors. However, these approaches have not been extended to the unknown-break-date case, and they would be unable to simultaneously detect type-1 instabilities if the data provide no evidence in favor of a change in the number of factors.

The model selection mechanism in this paper employs the adaptive group LASSO estimator (Tibshirani (1994), Zou (2006), and Yuan and Lin (2006)) of a high-dimensional 
sparse system. This sparse system unifies the factor structures and the specification of structural changes. Specifically, we consider penalized estimation of the factor loadings and changes in factor loadings in an augmented auxiliary model where the factors are replaced by a large number of orthonormal regressors. Consistent model selection is obtained by combining superefficient estimation of the zero components and consistent estimation of the nonzero components in the sparse system. Theoretical results in the paper provide bounds on the penalization tuning parameters for consistent model selection. A practical algorithm is suggested for empirical applications. In recent work, Bai and Liao (2012), Caner and Han (2012), and Lu and Su (2013) provide important results on shrinkage estimation of stable factor models. With observed regressors, Lee, Seo, and Shin (2012) and Qian and Su (2013) propose using shrinkage estimation to detect structural breaks. The main challenge in our paper is that the factor structure is both unobserved and unstable.

The remainder of this paper is organized as follows. Section 2 describes the factor model and the types of instabilities considered in this paper. The proposed shrinkage estimator and model selection procedure are presented in Section 3 under the assumption that the break date is known. Section 4 develops the asymptotic theory for our estimator and establishes the consistency of the model selection procedure. The selection of the tuning parameters as well as the practical implementation of the shrinkage estimation are addressed in Section 5. The extension to the case in which the break date is unknown is presented in Section 6 . The Monte Carlo results are reported in Section 7, and Section 8 contains the empirical application. Finally, Section 9 concludes. All proofs as well as additional simulation and empirical results are relegated to the Appendix.

\section{A Factor Model with Structural Break}

We observe panel data $\left\{X_{i t} \in R: i=1, \ldots, N, t=1, \ldots, T\right\}$. Let $X_{t}=\left(X_{1 t}, \ldots, X_{N t}\right)^{\prime} \in$ $R^{N \times 1}$ denote the observations at time period $t$. For $t=1, \ldots, T_{0}$, the observed $N$ series are driven by $r_{a}$ unobserved common factors. At time period $T_{0}$, the number of factors and/or the magnitude of the factor loadings may change. We assume that there are no further breaks after $T_{0}$. Using the break date $T_{0}$, we split the full sample into two stable subsamples: The first one contains the first $T_{0}$ observations of the $N$ series, and the second one contains the last $T_{1}=T-T_{0}$ observations of the $N$ series. In the remainder of this section, we introduce the (nonidentifiable) data generating process (DGP) and an identifiable version of the DGP. 
Moreover, we distinguish between two types of structural changes, the occurrence of which we will consistently estimate.

\subsection{The (Nonidentifiable) Data Generating Process}

The DGP before $T_{0}$ is

$$
X_{t}=\Lambda^{0} F_{t}^{0}+e_{t}, \text { for } t=1, \ldots, T_{0},
$$

where $\Lambda^{0} \in R^{N \times r_{a}}$ denotes the factor loadings and $e_{t} \in R^{N}$ denotes the idiosyncratic errors. Using matrix notation, we write

$$
X_{a}=F_{a} \Lambda^{0 \prime}+e_{a}
$$

where $X_{a}=\left(X_{1}, \ldots, X_{T_{0}}\right)^{\prime} \in R^{T_{0} \times N}, F_{a}=\left(F_{1}^{0}, \ldots, F_{T_{0}}^{0}\right)^{\prime} \in R^{T_{0} \times r_{a}}$, and $e_{a}=\left(e_{1}, \ldots, e_{T_{0}}\right)^{\prime} \in$ $R^{T_{0} \times N}$. The matrices $F_{a}$ and $\Lambda^{0}$ are both unknown and they are not separately identified.

To take into account the potential structural break in period $T_{0}$, we write the post-break DGP in matrix form as

$$
X_{b}=F_{b, 1}\left(\Lambda^{0}+\Gamma_{1}^{0}\right)^{\prime}+F_{b, 2} \Gamma_{2}^{0 \prime}+e_{b},
$$

where $X_{b}=\left(X_{T_{0}+1}, \ldots, X_{T}\right)^{\prime}, F_{b, 1}=\left(F_{T_{0}+1}^{0}, \ldots, F_{T}^{0}\right)^{\prime}, F_{b, 2}=\left(F_{T_{0}+1}^{*}, \ldots, F_{T}^{*}\right)^{\prime}$, and $e_{b}=$ $\left(e_{T_{0}+1}, \ldots, e_{T}\right)^{\prime}$. Here the $T_{1} \times r_{a}$ matrix $F_{b, 1}$ extends the pre-break factors to the post-break period, whereas the $T_{1} \times\left(r_{b}-r_{a}\right)$ matrix $F_{b, 2}$ collects the new factors that may emerge after the break. The matrix $\Gamma_{1}^{0}$ captures possible changes in the loadings of the pre-break factors $F_{t}^{0}$, whereas the matrix $\Gamma_{2}^{0}$ contains the loadings for the new factors $F_{t}^{*}$. The changes in the factor loadings are summarized in $\Gamma^{0}=\left(\Gamma_{1}^{0}, \Gamma_{2}^{0}\right)$. If the loadings of the old factors stay constant, then $\Gamma_{1}^{0}=0$. Likewise, in the absence of new factors $\Gamma_{2}^{0}=0$. After $T_{0}$, there are $r_{b}$ factors $F_{b}=\left(F_{b, 1}, F_{b, 2}\right)$ with factor loadings $\Psi^{0}=\left(\Lambda^{0}+\Gamma_{1}^{0}, \Gamma_{2}^{0}\right)$. Thus, the model in (2.3) can be equivalently written as

$$
X_{b}=F_{b} \Psi^{0 \prime}+e_{b}
$$

Throughout this paper, we use $C \in R$ to denote a generic positive constant. For $t>T_{0}$, let $\bar{F}_{t}^{0}=\left(F_{t}^{0 \prime}, F_{t}^{* \prime}\right)^{\prime} \in R^{r_{b}}$ denote the $r_{b}$ factors after the break. We assume the factors and their loadings satisfy Assumptions A and B below.

Assumption A. $\mathbb{E}\left[\left\|F_{t}^{0}\right\|^{4}\right] \leq C, \mathbb{E}\left[\left\|\bar{F}_{t}^{0}\right\|^{4}\right] \leq C$ and there exist positive definite matrices $\Sigma_{F}$ and $\Sigma_{\bar{F}}$ such that $T_{0}^{-1} \sum_{t=1}^{T_{0}} F_{t}^{0} F_{t}^{0 \prime}=\Sigma_{F}+O_{p}\left(T_{0}^{-1 / 2}\right)$ and $T_{1}^{-1} \sum_{t=T_{0}+1}^{T} \bar{F}_{t}^{0} \bar{F}_{t}^{0 \prime}=\Sigma_{\bar{F}}+$ $O_{p}\left(T_{1}^{-1 / 2}\right)$. 
Write $\Lambda^{0}=\left(\lambda_{1}^{0}, \ldots, \lambda_{N}^{0}\right)^{\prime}$, where $\lambda_{i}^{0} \in R^{r_{a} \times 1}$ is the factor loading for series $i$ before the break. Similarly, write $\Psi^{0}=\left(\psi_{1}^{0}, \ldots, \psi_{N}^{0}\right)^{\prime}$, where $\psi_{i}^{0} \in R^{r_{b} \times 1}$ is the factor loading for series $i$ after the break.

Assumption B. (i) $\left\|\lambda_{i}^{0}\right\| \leq C,\left\|\psi_{i}^{0}\right\| \leq C$ and there exist matrices $\Sigma_{\Lambda}, \Sigma_{\Psi}$ and $\Sigma_{\Lambda \Psi}$ such that $\left\|\Lambda^{0 \prime} \Lambda^{0} / N-\Sigma_{\Lambda}\right\| \rightarrow 0,\left\|\Psi^{0 \prime} \Psi^{0} / N-\Sigma_{\Psi}\right\| \rightarrow 0$, and $\left\|\Lambda^{0 \prime} \Psi^{0} / N-\Sigma_{\Lambda \Psi}\right\| \rightarrow 0$ as $N \rightarrow \infty$, where $\Sigma_{\Lambda}$ and $\Sigma_{\Psi}$ are positive definite. (ii) The matrices $\Sigma_{\Lambda} \Sigma_{F}$ and $\Sigma_{\Psi} \Sigma_{\bar{F}}$ both have distinct eigenvalues.

Assumptions A and B are analogous to Assumptions A and B of Bai and Ng (2002) with the modification to accommodate additional factors and changes of factor loadings at $T_{0}$. They ensure that all $r_{a}$ factors before the break and $r_{b}$ factors after the break make nontrivial contributions to the variance of the data. Assumption B(ii) is the same as Assumption G of Bai (2003). ${ }^{1}$

In the remainder of this paper, we assume $r_{b} \geq r_{a}$. If the application suggests that $r_{b} \leq r_{a}$, then labeling the subsample before $T_{0}$ as $X_{b}$ and the subsample after $T_{0}$ as $X_{a}$ maintains the validity of the proposed method. We distinguish between two types of instabilities:

$$
\begin{array}{ll}
\text { type- } 1 \text { change }: & r_{b}=r_{a} \text { and } \Gamma_{1}^{0} \neq 0 \\
\text { type- } 2 \text { change }: & r_{b}>r_{a} .
\end{array}
$$

Under a type-1 change, the number of factors is constant, but there is a change in the factor loadings. For a type-2 change, new factors appear in the model after $T_{0}$, while some of the loadings of the old factors also may change.

If the break date $T_{0}$ is known, the number of pre- and post-break factors $r_{a}$ and $r_{b}$ are identified and can be consistently estimated using existing methods (e.g., the model selection criteria proposed by Bai and $\mathrm{Ng}(2002))$. The strict inequality $r_{b}>r_{a}$ identifies type-2 instabilities without further assumptions on the DGP. To identify type-1 instabilities, further restrictions are necessary. Intuitively, the change is identifiable if either the space spanned by the factor loadings or the scaling of the factor loadings changes. Formally, for any square matrix $A$, we use $\rho_{\ell}(A)$ to denote its $\ell$-th largest eigenvalue. Define a $\left(r_{a}+r_{b}\right) \times\left(r_{a}+r_{b}\right)$

\footnotetext{
${ }^{1}$ Assumption A is sufficient for the identification conditions in Assumption ID below. It is also one of the sufficient conditions for consistent model selection with a known break date. For consistent model selection with an unknown break date, Assumption A is strengthened to Assumption A* in Section 6.1.
} 
augmented covariance matrix

$$
\Sigma_{\Lambda \Psi}^{+}=\left[\begin{array}{cc}
\Sigma_{\Lambda} & \Sigma_{\Lambda \Psi} \\
\Sigma_{\Lambda \Psi}^{\prime} & \Sigma_{\Psi}
\end{array}\right] .
$$

The following assumption, stated in terms of the coefficients of the DGP in (2.2) and (2.3), is sufficient for identifying type-1 structural instabilities.

Assumption ID. One of the following two conditions holds:

(i) $\operatorname{rank}\left(\Sigma_{\Lambda \Psi}^{+}\right)>r_{a}$;

(ii) $\rho_{\ell}\left(\Sigma_{F} \Sigma_{\Lambda}\right) \neq \rho_{\ell}\left(\Sigma_{\bar{F}} \Sigma_{\Psi}\right)$ for some $\ell \leq r_{a}$.

Assumption ID(i) holds if and only if $\Lambda^{0}$ and $\Psi^{0}$ do not span the same column space asymptotically. Assumption ID(ii) focuses on the scaling of the loadings and provides an alternative identification condition through the eigenvalues of $\Sigma_{\Lambda} \Sigma_{F}$ and $\Sigma_{\Psi} \Sigma_{\bar{F}}$. This condition does not put restrictions on the asymptotic column spaces generated by the factor loadings.

\subsection{An Identifiable Version of the DGP}

The factors and their loadings in (2.2) and (2.4) are not separately identified. In order to develop an estimation theory for the factor model, we have to impose normalization restrictions. This normalization also helps to further clarify identifiable type- 1 structural changes. Because our estimation will be based on principal-components analysis, we normalize the factors to have an identity covariance matrix and the vectors of factor loadings to be orthogonal and sorted according to length. ${ }^{2}$

Let $\Sigma_{a}=\Lambda^{0 \prime} \Lambda^{0} / N \in R^{r_{a} \times r_{a}}$, let $\Sigma_{a}^{1 / 2}$ be the Cholesky factor of $\Sigma_{a}$, and let $\Upsilon_{a}$ be a matrix of orthonormal eigenvectors such that

$$
\Upsilon_{a}^{\prime}\left(\Sigma_{a}^{1 / 2}\right)^{\prime} \Sigma_{F} \Sigma_{a}^{1 / 2} \Upsilon_{a}=V_{a}
$$

where $V_{a}$ is a diagonal matrix of eigenvalues, ordered from largest to smallest. Note that by Assumptions A and B, the matrix $\left(\Sigma_{a}^{1 / 2}\right)^{\prime} \Sigma_{F} \Sigma_{a}^{1 / 2}$ has positive and distinct eigenvalues with large $N$, which means that (2.7) holds for large $N$. Now define the transformation matrix

$$
R_{a}=\Sigma_{a}^{1 / 2} \Upsilon_{a} V_{a}^{-1 / 2}
$$

\footnotetext{
${ }^{2}$ In addition, the signs of the factors and loadings need to be normalized. However, because this sign normalizaton is immaterial for our analysis, we do not provide further details.
} 
We can rewrite the DGP before $T_{0}$ as

$$
X_{a}=F_{a} R_{a} R_{a}^{-1} \Lambda^{0 \prime}+e_{a}=F_{a}^{R} \Lambda^{R \prime}+e_{a},
$$

where $F_{a}^{R}=F_{a} R_{a}$ and $\Lambda^{R}=\Lambda^{0}\left(R_{a}^{-1}\right)^{\prime}$. For the post-break DGP, we let $\Sigma_{b}=\Psi^{0 /} \Psi^{0} / N \in$ $R^{r_{b} \times r_{b}}$, substitute $\Sigma_{F}$ in (2.7) by $\Sigma_{\bar{F}}$, and otherwise replace $a$ subscripts by $b$ subscripts. The second transformation matrix $R_{b}$ is defined as

$$
R_{b}=\Sigma_{b}^{1 / 2} \Upsilon_{b} V_{b}^{-1 / 2}
$$

such that the second DGP can be rewritten s $^{3}$

$$
X_{b}=F_{b} R_{b} R_{b}^{-1} \Psi^{0 \prime}+e_{b}=F_{b}^{R} \Psi^{R \prime}+e_{b}
$$

Assumption ID(i) implies that the column spaces of $\Lambda^{R}$ and $\Psi^{R}$ in (2.9) and (2.11) are different, whereas Assumption ID(ii) translates into $V_{a} \neq V_{b}$. Note that our normalization interprets changes in the law of motion of the factors $F_{a}$ and $F_{b}$ as changes in the loadings $\Lambda^{R}$ and $\Psi^{R}$. For example, consider a DGP with $r_{a}=r_{b}=1$, constant factor loadings $\Lambda=\Psi$, and a break in the persistence of the factor, which follows an $\operatorname{AR}(1)$ process $F_{t}=\rho_{a} F_{t-1}+\varepsilon_{t}$ for $t \leq T_{0}$ and $F_{t}=\rho_{b} F_{t-1}+\varepsilon_{t}$ for $T>T_{0}$, where $\varepsilon_{t} \sim$ i.i.d. $N(0,1)$ for all $t$. The change of the autocorrelation of $F_{t}$ from $\rho_{a}$ to $\rho_{b}$ in our setting translates into a change of the transformed factor loadings from $\Lambda^{R}=\Lambda / \sqrt{1-\rho_{a}^{2}}$ to $\Psi^{R}=\Lambda / \sqrt{1-\rho_{b}^{2}}$. This leads to $V_{b}=V_{a}\left(1-\rho_{b}^{2}\right) /\left(1-\rho_{a}^{2}\right)$.

\subsection{A Useful Decomposition of Structural Changes}

The (nonidentifiable) DGP in (2.2) and (2.3) provides a natural decomposition of type-2 structural changes into changes resulting from the new factors, $F_{b, 2} \Gamma_{2}^{0^{\prime}}$, and changes associated with the effect of the extended versions of the old factors, $F_{b, 1} \Gamma_{1}^{0^{\prime}}$. We can mechanically rewrite the normalized version of the post-break DGP in (2.11) as

$$
X_{b}=F_{b}^{R} \Psi^{R \prime}+e_{b}=F_{b, 1}^{R}\left(\Lambda^{R}+\Gamma_{1}^{R}\right)^{\prime}+F_{b, 2}^{R} \Gamma_{2}^{R^{\prime}}+e_{b},
$$

\footnotetext{
${ }^{3}$ It can be verified that the transformation induces the desired normalization. For the pre-break period, using Assumption A and the fact that $\Upsilon_{a}$ is a finite matrix, we have

$$
T_{a}^{-1} F_{a}^{R \prime} F_{a}^{R}=V_{a}^{-1 / 2} \Upsilon_{a}^{\prime} \Sigma_{a}^{1 / 2} \Sigma_{F} \Sigma_{a}^{1 / 2} \Upsilon_{a} V_{a}^{-1 / 2}+O_{p}\left(T_{a}^{-1 / 2}\right)=I_{r_{a} \times r_{a}}+O_{p}\left(T_{a}^{-1 / 2}\right) .
$$

Moreover, by definition of $\Sigma_{a}, N^{-1} \Lambda^{R \prime} \Lambda^{R}=V_{a}^{1 / 2} \Upsilon_{a}^{\prime} \Sigma_{a}^{-1 / 2} \Sigma_{a}\left(\Sigma_{a}^{-1 / 2}\right)^{\prime} \Upsilon_{a} V_{a}^{1 / 2}=V_{a}$, which is a diagonal matrix, as desired.
} 
where $F_{b}^{R}=\left(F_{b, 1}^{R}, F_{b, 2}^{R}\right)$, and we define $\Gamma^{R}=\left(\Gamma_{1}^{R}, \Gamma_{2}^{R}\right)$. However, the components $F_{b, 1}^{R} \Gamma_{1}^{R^{\prime}}$ and $F_{b, 2}^{R} \Gamma_{2}^{R^{\prime}}$ are difficult to interpret because after imposing the normalization, there is no sense in which $F_{b, 1}$ can be viewed as the post-break extension of $F_{a}$.

Nonetheless, in empirical applications, it is interesting and useful to decompose type-2 changes into the contribution of the new factors and changes in the effects of old factors. To do so, we construct an $r_{b} \times r_{a}$ matrix with orthogonal columns by maximizing the correlation between the old normalized loadings $\Lambda^{R}$ and the new loadings $\Psi^{R} \Omega_{a}$ :

$$
\Omega_{a}=\operatorname{argmax}_{\tilde{\Omega}_{a} \in \mathcal{O}} \operatorname{tr}\left[\Lambda^{R^{\prime}} \Psi^{R} \tilde{\Omega}_{a}\right]
$$

where $\mathcal{O}$ is the class of $r_{b} \times r_{a}$ matrices with orthonormal columns. The solution is given by (see Cliff (1966)) $\Omega_{a}=V U^{\prime}$, where $V$ is an $r_{b} \times r_{a}$ and $U$ an $r_{a} \times r_{a}$ orthogonal matrix obtained from the singular value decomposition $\Lambda^{R^{\prime}} \Psi^{R}=U D V^{\prime}$. Let $\Omega_{\perp}$ be the null space of $\Omega_{a}^{\prime}$ and define $\Omega=\left(\Omega_{a}, \Omega_{\perp}\right)$. Moreover, define the rotated loadings and factors $F_{b}^{R \Omega}=F^{R} \Omega$ and $\Psi^{R \Omega}=\Psi^{R} \Omega$. This rotation preserves the normalization of the factors, i.e., $F_{b}^{R \Omega^{\prime}} F_{b}^{R \Omega} / T_{b}=I$. Partitioning $F_{b}^{R \Omega}=\left(F_{b, 1}^{R \Omega}, F_{b, 2}^{R \Omega}\right)$ and $\Psi^{R \Omega}=\left(\Psi_{1}^{R \Omega}, \Psi_{2}^{R \Omega}\right)$, we can decompose $X_{b}$ as follows:

$$
X_{b}=F_{b}^{R} \Omega \Omega^{\prime} \Psi^{R^{\prime}}+e_{b}=\underbrace{F_{b, 1}^{R \Omega} \Lambda^{R^{\prime}}}_{\text {old loadings }}+\underbrace{F_{b, 1}^{R \Omega}\left(\Psi_{1}^{R \Omega}-\Lambda^{R}\right)^{\prime}}_{\text {change in loadings }}+\underbrace{F_{b, 2}^{R \Omega} \Psi_{2}^{R \Omega^{\prime}}}_{\text {new factor }}+e_{b} .
$$

\subsection{Model Classes}

The main contribution of this paper is to develop a procedure that consistently detects the occurrence of type- 1 and type- 2 structural changes. Let $\mathcal{S}_{0} \in\{0,1\}$ be a binary variable such that $\mathcal{S}_{0}=0$ indicates that there is no structural instability (i.e., $\Gamma^{(0)}=0$ in $(2.3)$ and $\left.r_{a}=r_{b}\right)$. If $\mathcal{S}_{0}=1$ and $r_{a}=r_{b}$, then the DGP exhibits a type- 1 instability. Finally, $\mathcal{S}_{0}=1$ and $r_{a}<r_{b}$ corresponds to a type- 2 instability. For the remainder of this paper, we refer to a model as a collection of DGPs that are associated with the triplet

$$
\mathcal{M}_{0}=\left(r_{a}, r_{b}, \mathcal{S}_{0}\right)
$$

We propose a consistent model selection procedure for $\mathcal{M}_{0}$ based on the simultaneous estimation of $r_{a}, r_{b}$, and $\mathcal{S}_{0}$. For the consistent determination of $\mathcal{M}_{0}$, it suffices to estimate the normalized version of the factor model in (2.9) and (2.11), because $\Gamma^{0}=0$ if and only if $\Gamma^{R}=0$, where $\Gamma_{1}^{R}$ and $\Gamma_{2}^{R}$ are (implicitly) defined in (2.12). Moreover, $\Gamma_{2}^{0}=0$ if and only if $\Gamma_{2}^{R}=0$. 
Our procedure differs from the existing methods in two very important dimensions. First, our method not only detects structural instabilities but also automatically determines their type. Second, to detect instabilities in factor loadings, our method does not require knowledge of the number of factors before and/or after the break. Instead, it determines the preand post-break factors structures simultaneously.

\section{Model Selection with Known Break Date}

In this section, we assume that the date of the potential structural break, $T_{0}$, is known. We divide the full sample into a pre-break and a post-break subsample. Let $T_{a}$ and $T_{b}$ denote the number of periods in the two subsamples, respectively. With a known break date, $T_{a}=T_{0}$ and $T_{b}=T_{1}$. Since we treat the number of factors as unknown, we define $k \geq r_{b}$ to be the number of potential factors. In order to motivate the criterion function in the shrinkage estimation, we rewrite the normalized DGP in (2.9) and (2.11) as the following augmented system:

$$
\begin{aligned}
& X_{a}=\left[\begin{array}{lll}
F_{a}^{R} & F_{a, 1}^{R \perp} & F_{a, 2}^{R \perp}
\end{array}\right]\left[\begin{array}{c}
\Lambda^{R \prime} \\
0_{\left(r_{b}-r_{a}\right) \times N} \\
0_{\left(k-r_{b}\right) \times N}
\end{array}\right]+e_{a}=F_{a}^{R+}\left(\Lambda^{R+}\right)^{\prime}+e_{a} . \\
& X_{b}=\left[\begin{array}{lll}
F_{b, 1}^{R} & F_{b, 2}^{R} & F_{b}^{R \perp}
\end{array}\right]\left[\begin{array}{c}
\Lambda^{R \prime}+\Gamma_{1}^{R \prime} \\
\Gamma_{2}^{R \prime} \\
0_{\left(k-r_{b}\right) \times N}
\end{array}\right]+e_{b}=F_{b}^{R+}\left(\Lambda^{R+}+\Gamma^{R+}\right)^{\prime}+e_{b} .
\end{aligned}
$$

Here, $F_{a}^{R \perp}$ denotes a $T \times\left(k-r_{a}\right)$ orthogonal complement of $F_{a}^{R}$. We partition $F_{a}^{R \perp}$ into $T \times\left(r_{b}-r_{a}\right)$ and $T \times\left(k-r_{b}\right)$ submatrices $F_{a, 1}^{R \perp}$ and $F_{a, 2}^{R \perp}$. Likewise, $F_{b}^{R \perp}$ is an orthogonal complement of $F_{b}^{R}$. Below, we call $F_{a}^{R}$ and $F_{b}^{R}$ the "true" and $F_{a}^{R \perp}$ and $F_{b}^{R \perp}$ the irrelevant factors. In the augmented model $(3.1), \Lambda^{R+}$ and $\left(\Lambda^{R+}+\Gamma^{R+}\right)$ are the factor loadings before and after the break, respectively. Estimating the number of factors and detecting instability in factor loadings can be executed simultaneously in (3.1), because they are equivalent to consistent selection of the zero and and nonzero components in $\Lambda^{R+}$ and $\Gamma^{R+}$. Hence, for consistent model selection, it is key to obtain estimators that can consistently distinguish zeros from nonzeros in $\Lambda^{R+}$ and $\Gamma^{R+}$. The shrinkage estimator proposed below is designed to achieve such consistency. 


\subsection{Estimation Objective Function}

The $k$ potential factors are estimated by the principal component estimator in each subsample. Specifically, for subsample $j \in\{a, b\}$, let $\widetilde{F}_{j} \in R^{T_{j} \times k}$ be the orthonormalized eigenvectors of $\left(N T_{j}\right)^{-1} X_{j} X_{j}^{\prime}$ associated with its first $k$ largest eigenvalues. For both subsamples, estimating an overfitted model with $k$ factors gives the unrestricted least square estimators of the factor loading matrices $\widetilde{\Lambda}_{L S}=T_{a}^{-1} X_{a}^{\prime} \widetilde{F}_{a}, \widetilde{\Psi}_{L S}=T_{b}^{-1} X_{b}^{\prime} \widetilde{F}_{b}$ and $\widetilde{\Gamma}_{L S}=\widetilde{\Psi}_{L S}-\widetilde{\Lambda}_{L S}$.

Given $\widetilde{F}_{a}$ and $\widetilde{F}_{b}$, we propose shrinkage estimators of $\Lambda^{R+}$ and $\Gamma^{R+}$ by minimizing a PLS criterion function:

$$
(\widehat{\Lambda}, \widehat{\Gamma})=\underset{\Lambda \in R^{N \times k}, \Gamma \in R^{N \times k}}{\arg \min }\left[M(\Lambda, \Gamma)+P_{1}(\Lambda)+P_{2}(\Gamma)\right]
$$

where

$$
\begin{aligned}
M(\Lambda, \Gamma) & =(N T)^{-1}\left[\left\|X_{a}-\widetilde{F}_{a} \Lambda^{\prime}\right\|^{2}+\left\|X_{b}-\widetilde{F}_{b}(\Lambda+\Gamma)^{\prime}\right\|^{2}\right] \\
P_{1}(\Lambda) & =\alpha_{N T} \sum_{\ell=1}^{k} \omega_{\ell}^{\lambda}\left\|\Lambda_{\ell}\right\| \text { and } P_{2}(\Gamma)=\beta_{N T} \sum_{\ell=1}^{k} \omega_{\ell}^{\gamma}\left\|\Gamma_{\ell}\right\|,
\end{aligned}
$$

$\Lambda_{\ell}$ and $\Gamma_{\ell}$ are the $\ell$-th column of $\Lambda$ and $\Gamma$, respectively, $\alpha_{N T}$ and $\beta_{N T}$ are two sequences of positive constants that depend on $N$ and $T$, and $\omega_{\ell}^{\lambda}$ and $\omega_{\ell}^{\gamma}$ are data-dependent weights defined as:

$$
\begin{aligned}
& \omega_{\ell}^{\lambda}=\left(N^{-1}\left\|\widetilde{\Lambda}_{\ell}\right\|^{2} \mathcal{I}_{\left\{\widetilde{\Lambda}_{\ell} \neq 0_{N \times 1}\right\}}+N^{-1}\left\|\widetilde{\Lambda}_{\ell, L S}\right\|^{2} \mathcal{I}_{\left\{\widetilde{\Lambda}_{\ell}=0_{N \times 1}\right\}}\right)^{-d}, \\
& \omega_{\ell}^{\gamma}=\left(N^{-1}\left\|\widetilde{\Gamma}_{\ell}\right\|^{2} \mathcal{I}_{\left\{\widetilde{\Gamma}_{\ell} \neq 0_{N \times 1}\right\}}+N^{-1}\left\|\widetilde{\Gamma}_{\ell, L S}\right\|^{2} \mathcal{I}_{\left\{\widetilde{\Gamma}_{\ell}=0_{N \times 1}\right\}}\right)^{-d} .
\end{aligned}
$$

Here $\mathcal{I}_{\{x=a\}}$ is the indicator function that is equal to one if $x=a$ and equal to zero otherwise; $d$ is a positive constant; and $\widetilde{\Lambda} \in R^{N \times k}$ and $\widetilde{\Gamma} \in R^{N \times k}$ are some preliminary estimators of $\Lambda^{+}$and $\Gamma^{+}$, where the $\ell$ subscript denotes the $\ell$-th column of the matrices. The simplest preliminary estimator available is the unrestricted least square estimator (i.e., $\widetilde{\Lambda}=\widetilde{\Lambda}_{L S}$ and $\left.\widetilde{\Gamma}_{L S}=\widetilde{\Gamma}_{L S}\right)$. An alternative preliminary estimator can be a shrinkage estimator that is based on a rough choice of tuning parameters. Since such a shrinkage estimator may set some of the columns of $\tilde{\Lambda}$ and $\tilde{\Gamma}$ equal to zero, we use the indicator functions to replace zero columns with the corresponding columns of the unrestricted least square estimators. This model selection procedure is easy to compute because it is a convex optimization problem after the principal components are used for dimension reduction. 
In this adaptive estimation, the data-dependent weights $\omega_{\ell}^{\lambda}$ and $\omega_{\ell}^{\gamma}$ are designed to differentiate the zero columns of $\Lambda^{R+}$ and $\Gamma^{R+}$ from the nonzero columns. It is easy to obtain preliminary estimators such that $\left\|\widetilde{\Lambda}_{\ell}\right\| \rightarrow_{p} 0$ if and only if the $\ell$-th column of $\Lambda^{R+}$ is zero and $\left\|\widetilde{\Gamma}_{\ell}\right\| \rightarrow_{p} 0$ if and only if the $\ell$-th column of $\Gamma^{R+}$ is zero. The unrestricted least square estimator is one simple example. If such requirement is satisfied by the preliminary estimator, we expect $N^{-1}\left\|\widetilde{\Lambda}_{\ell}\right\|$ to converge to a positive constant for $\ell \leq r_{a}$ and to converge to zero for $\ell>r_{a}$. In the latter case, $\omega_{\ell}^{\lambda}$ diverges to infinity, which delivers strong penalization in the shrinkage estimation (3.2) to the estimators of the zero columns in $\Lambda^{0}$. The weights, $\omega_{\ell}^{\gamma}$, have similar effects on the estimation of $\Gamma^{+}$.

The penalty functions $P_{1}(\Lambda)$ and $P_{2}(\Gamma)$, defined in terms of the column norms $\left\|\Lambda_{\ell}\right\|$ and $\left\|\Gamma_{\ell}\right\|$, are group-LASSO penalties (cf., Yuan and Lin (2006)). A group-LASSO estimator either sets all the elements in a group equal to zero or estimates them as nonzeros altogether. This feature is particularly useful for large-scale factor models because the irrelevant factors have zero factor loadings for all series. As such, the group-LASSO estimator automatically controls the group-wise model-selection error, which is challenging if the model-selection is performed sequentially.

\subsection{Model Selection}

Model selection for $\mathcal{M}_{0}$ is based on the column norms of $\widehat{\Lambda}$ and $\widehat{\Gamma}$. The estimators of $r_{a}$ and $r_{b}$ are

$$
\begin{aligned}
& \widehat{r}_{a}=\min \mathcal{J}_{a}, \text { where } \mathcal{J}_{a}=\left\{j:\left\|\widehat{\Lambda}_{\ell}\right\|^{2}=0 \text { for all } \ell>j\right\} \\
& \widehat{r}_{b}=\max \left(\min \mathcal{J}_{b}, \widehat{r}_{a}\right) \text {, where } \mathcal{J}_{b}=\left\{j:\left\|\widehat{\Gamma}_{\ell}\right\|^{2}=0 \text { for all } \ell>j\right\} .
\end{aligned}
$$

Note that $\min \mathcal{J}_{a}$ is the last nonzero column of $\widehat{\Lambda}$ and $\min \mathcal{J}_{b}$ is the last nonzero column of $\widehat{\Gamma}$. The estimator of $\mathcal{S}_{0}$ is

$$
\widehat{\mathcal{S}}=\left\{\begin{array}{cc}
0 & \text { if } \widehat{\Gamma}=0, \\
1 & \text { otherwise }
\end{array}\right.
$$

The procedure selects a model with no structural instability if $\widehat{\mathcal{S}}=0$. When $\widehat{\mathcal{S}} \neq 0$, the change is type-1 if $\widehat{r}_{b}=\widehat{r}_{a}$ and it is type-2 if $\widehat{r}_{b}>\widehat{r}_{a} .{ }^{4}$ In all cases, we not only detect the

\footnotetext{
${ }^{4}$ If there is a type- 2 change, the first $\widehat{r}_{a}$ columns of $\widehat{\Gamma}$ do not provide any implications on the stability of the "original factor loadings" in the first subsample, because they involve a rotation of all factor loadings, including the new ones.
} 
instabilities and identify their sources but also simultaneously estimate the number of factors before and after the break. In sum, the model selected by the shrinkage estimator is

$$
\widehat{\mathcal{M}}=\left(\widehat{r}_{a}, \widehat{r}_{b}, \widehat{\mathcal{S}}\right) .
$$

In the following sections, we show that

$$
\operatorname{Pr}\left(\widehat{\mathcal{M}}=\mathcal{M}_{0}\right) \rightarrow 1 \text { as } N, T \rightarrow \infty
$$

provided that the tuning parameters $\alpha_{N T}$ and $\beta_{N T}$ are chosen within the bounds specified below.

\subsection{Estimation of Factor Loadings}

In applications such as forecasting both the dimension of the factor space and the values of the factor loadings are of interest. If $\hat{\mathcal{S}}=0$ (i.e., there is no evidence of a structural instability), then we recommend to reestimate the factors and their loadings using the full sample to improve efficiency and reduce the bias introduced by penalization. In this case, let $\widetilde{F} \in R^{T \times \widehat{r}_{a}}$ be the orthonormalized left eigenvectors of $(N T)^{-1} X X^{\prime}$ associated with its first $\widehat{r}_{a}$ largest eigenvalues. The factor loading for the full sample is $\widehat{\Psi}_{F}=T^{-1} X^{\prime} \widetilde{F}$.

If, on the other hand, there is evidence of a structural break, $\hat{\mathcal{S}}=1$, then one could either use the pre- and post-break shrinkage estimators $\widehat{\Lambda}$ and $\widehat{\Lambda}+\widehat{\Gamma}$ of the factor loadings or re-estimate the factor model conditional on the selected number of factors $\hat{r}_{a}$ and $\hat{r}_{b}$. We call this latter estimator a post-model-selection (PMS) estimator. It is formally defined as

$$
\widehat{\Lambda}_{P M S}=\left(\bar{\Lambda}, 0_{\Lambda}\right), \widehat{\Psi}_{P M S}=\left(\bar{\Psi}, 0_{\Psi}\right) \text { and } \widehat{\Gamma}_{P M S}=\widehat{\Psi}_{P M S}-\widehat{\Lambda}_{P M S}
$$

where $\bar{\Lambda}$ denotes the first $\widehat{r}_{a}$ columns of $\widetilde{\Lambda}_{L S}, \bar{\Psi}$ denotes the first $\widehat{r}_{b}$ columns of $\widetilde{\Psi}_{L S}, 0_{\Lambda}$ is a $N \times\left(k-\widehat{r}_{a}\right)$ zero matrix, and $0_{\Psi}$ is a $N \times\left(k-\widehat{r}_{b}\right)$ zero matrix. For the first $\widehat{r}_{a}$ columns, the PMS estimator is identical to the unrestricted least square estimator because the columns of $\widetilde{F}_{a}$ are orthogonal by construction. The same argument applies to $\widehat{\Psi}_{L S}$. In finite samples, the penalization on the nonzero columns may further reduce the variance of the shrinkage estimator, but at the same time, it introduces extra bias. Whether this feature of the shrinkage estimator is preferable to the PMS estimator depends on the specific bias-variance trade-off, and we provide some simulation evidence in Section 7. For both the shrinkage estimators and the PMS estimators of the factor loadings, their corresponding factor estimators are $\widetilde{F}_{a}$ for the first subsample and $\widetilde{F}_{b}$ for the second subsample. 


\section{Asymptotic Theory}

This section establishes the large sample properties of the shrinkage estimators $(\widehat{\Lambda}, \widehat{\Gamma})$ and shows the consistency of the proposed model selection procedure when the break date is known. We begin by stating additional assumptions.

\subsection{Additional Assumptions}

Suppose $T_{0} / T \rightarrow \tau_{0}$ for some constant $\tau_{0} \in(0,1)$ as $T \rightarrow \infty$. We assume the following assumptions in addition to Assumptions $\mathrm{A}$ and $\mathrm{B}$. Let $e=\left[e_{1}, \ldots, e_{T}\right] \in R^{N \times T}$ be the matrix of idiosyncratic errors and $e_{i t}$ denote the $(i, t)$ element of $e$ that is associated with series $i$ in period $t$.

Assumption C. (i). $\mathbb{E}\left[e_{i t}\right]=0, \mathbb{E}\left[\left|e_{i t}\right|^{8}\right] \leq C$;

(ii). $\mathbb{E}\left[N^{-1} \sum_{i=1}^{N} e_{i s} e_{i t}\right]=\sigma_{N}(s, t),\left|\sigma_{N}(s, s)\right| \leq C$ for all $s, T^{-1} \sum_{s=1}^{T} \sum_{t=1}^{T}\left|\sigma_{N}(s, t)\right| \leq C$;

(iii). $\mathbb{E}\left[e_{i t} e_{j t}\right]=\tau_{i j, t}$ with $\left|\tau_{i j, t}\right| \leq\left|\tau_{i j}\right|$ for some $\tau_{i j}$ and for all $t$, and $N^{-1} \sum_{i=1}^{N} \sum_{j=1}^{N}\left|\tau_{i j}\right| \leq C$;

(iv). $\mathbb{E}\left[e_{i t} e_{j s}\right]=\tau_{i j, t s}$ and $(N T)^{-1} \sum_{i=1}^{N} \sum_{j=1}^{N} \sum_{t=1}^{T} \sum_{s=1}^{T}\left|\tau_{i j, t s}\right| \leq C$;

(v). For every $(t, s), \mathbb{E}\left[\left|N^{-1 / 2} \sum_{i=1}^{N}\left[e_{i s} e_{i t}-\mathbb{E}\left[e_{i s} e_{i t}\right]\right]\right|^{4}\right] \leq C$;

(vi). $\rho_{1}\left((N T)^{-1} e_{a} e_{a}^{\prime}\right)=O_{p}\left(\max \left[N^{-1}, T^{-1}\right]\right)$ and $\rho_{1}\left((N T)^{-1} e_{b} e_{b}^{\prime}\right)=O_{p}\left(\max \left[N^{-1}, T^{-1}\right]\right)$.

Assumption D. $\mathbb{E}\left[N^{-1} \sum_{i=1}^{N}\left\|T^{-1 / 2}\left(\sum_{t=1}^{T_{0}} F_{t}^{0} e_{i t}+\sum_{t=T_{0}+1}^{T} \bar{F}_{t}^{0} e_{i t}\right)\right\|^{2}\right] \leq C$.

Assumptions C and D are analogous to Assumptions C and D of Bai and Ng (2002). Assumption $\mathrm{C}$ allows for time-series and cross-sectional weak dependence in the idiosyncratic errors. Assumption $\mathrm{C}(\mathrm{vi})$ or a similar condition is needed for the consistent selection of the number of factors (see Amengual and Watson (2007)). Assumption D allows for weak dependence between the factors and the idiosyncratic errors.

Define $C_{N T}=\min \left(T^{1 / 2}, N^{1 / 2}\right)$, where $C_{N T}$ is the convergence rate of the unrestricted least square estimator in Bai and Ng (2002). Assumptions P1 and P2 below are highlevel conditions on the stochastic order of the preliminary estimators. They are useful in studying the asymptotic properties of the data-dependent weights $\omega_{\ell}^{\lambda}$ and $\omega_{\ell}^{\gamma}$ defined in (3.4). In practice, we consider the least square estimators $\widetilde{\Lambda}_{L S}$ and $\widetilde{\Gamma}_{L S}$, as well as shrinkage estimators as preliminary estimators (see Section 5 for details).

Assumption P1. The preliminary estimators $\widetilde{\Lambda}$ and $\widetilde{\Gamma}$ satisfy 
(i) $\operatorname{Pr}\left(N^{-1}|| \widetilde{\Lambda}_{\ell} \|^{2} \geq C\right) \rightarrow 1$ for $\ell=1, \ldots, r_{a}, N^{-1}\left\|\widetilde{\Lambda}_{\ell}\right\|^{2}=O_{p}\left(C_{N T}^{-2}\right)$ for $\ell=r_{a}+1, \ldots, k$;

(ii) If $\Gamma^{0} \neq 0, \operatorname{Pr}\left(N^{-1}|| \widetilde{\Gamma}_{\ell} \|^{2} \geq C\right) \rightarrow 1$ for $\ell=1, \ldots, r_{b}, N^{-1}\left\|\widetilde{\Gamma}_{\ell}\right\|^{2}=O_{p}\left(C_{N T}^{-2}\right)$ for $\ell=$ $r_{b}+1, \ldots, k$;

(iii) If $\Gamma^{0}=0, N^{-1}|| \widetilde{\Gamma}_{\ell} \|^{2}=O_{p}\left(C_{N T}^{-2}\right)$ for $\ell=1, \ldots, k$.

Assumption P2. Assumption P1 holds with $\widetilde{\Lambda}=\widetilde{\Lambda}_{L S}$ and $\widetilde{\Gamma}=\widetilde{\Gamma}_{L S}$.

Assumption $\mathrm{P} 1$ is imposed on any preliminary estimators of $\Lambda^{R}$ and $\Gamma^{R}$. If the preliminary estimators are different from $\widetilde{\Lambda}_{L S}$ and $\widetilde{\Gamma}_{L S}$, Assumption P2 is still necessary because $\omega_{\ell}^{\lambda}$ and $\omega_{\ell}^{\gamma}$ depend on $\widetilde{\Lambda}_{L S}$ and $\widetilde{\Gamma}_{L S}$ whenever $\widetilde{\Lambda}$ or $\widetilde{\Gamma}$ has zero columns. Note that $\widetilde{\Lambda}_{\ell}=0$ is a special case of $N^{-1}|| \widetilde{\Lambda}_{\ell} \|^{2}=O_{p}\left(C_{N T}^{-2}\right)$ in Assumption P1, and the same argument applies to $\widetilde{\Gamma}_{\ell}$.

Under the conditions in Assumption P1, the columns of the preliminary estimators are divided into two categories. For the first category, $\operatorname{Pr}\left(N^{-1}\left\|\widetilde{\Lambda}_{\ell}\right\|^{2} \geq C\right) \rightarrow 1$ and $\operatorname{Pr}\left(N^{-1}|| \widetilde{\Gamma}_{\ell} \|^{2} \geq C\right) \rightarrow 1$ such that the data-dependent weights, $\omega_{\ell}^{\lambda}$ and $\omega_{\ell}^{\gamma}$, are stochastically bounded. For the second category, $N^{-1}\left\|\widetilde{\Lambda}_{\ell}\right\|^{2}=O_{p}\left(C_{N T}^{-2}\right)$ and $N^{-1}\left\|\widetilde{\Gamma}_{\ell}\right\|^{2}=O_{p}\left(C_{N T}^{-2}\right)$, which implies that $\omega_{\ell}^{\lambda}$ and $\omega_{\ell}^{\gamma}$ diverge in probability faster than $C_{N T}^{2 d}$. These large penalties in the second category yield shrinkage estimators that are equal to 0 w.p.a.1.

While the data-dependent weights $\omega_{\ell}^{\lambda}$ and $\omega_{\ell}^{\gamma}$ determine the relative penalties of different columns of factor loadings, the tuning parameters $\alpha_{N T}$ and $\beta_{N T}$ determine the overall penalization. We make the following assumptions about the rates at which the tuning parameters vanish asymptotically.

Assumption T. The tuning parameters $\alpha_{N T}$ and $\beta_{N T}$ satisfy

(i) $\alpha_{N T}=O\left(N^{-1 / 2} C_{N T}^{-1}\right)$ and $\beta_{N T}=O\left(N^{-1 / 2} C_{N T}^{-1}\right)$;

(ii) $N^{-1 / 2} C_{N T}^{-(2 d+1)}=o\left(\alpha_{N T}\right)$ and $N^{-1 / 2} C_{N T}^{-(2 d+1)}=o\left(\beta_{N T}\right)$.

Assumption T imposes bounds on the tuning parameters $\alpha_{N T}$ and $\beta_{N T}$. These bounds control the magnitudes of penalization on all columns and are designed for consistent model selection. The upper bound in Assumption T(i) ensures that if the data-dependent weights $\omega_{\ell}^{\lambda}$ and $\omega_{\ell}^{\gamma}$ are stochastically bounded, the penalties on the nonzero columns are small such that the shrinkage bias is negligible asymptotically. On the other hand, we aim to shrink the estimators of zero columns to zero. For this purpose, the lower bound in Assumption $\mathrm{T}$ (ii) requires that the tuning parameters $\alpha_{N T}$ and $\beta_{N T}$ converge to zero not too fast. The upper bound is larger than the lower bound provided that $d$ is positive. In the simulation 
and empirical application, we use $d=2$. Under the theoretical guidance by Assumption T, we discuss the practical choice of $\alpha_{N T}$ and $\beta_{N T}$ in Section 5.

\subsection{Asymptotic Behavior of the PLS Estimator}

We begin by defining the asymptotic limits of the PLS estimators $\widehat{\Lambda}$ and $\widehat{\Gamma}$. The estimators converge to the coefficients of the normalized version of the DGP in (2.9) and (2.11). The transformation matrices $R_{a}$ and $R_{b}$, defined in (2.8) and (2.10), that were used to normalize the DGP are related to, but essentially different from, their counterparts considered in the literature, such as those in Bai and Ng (2002) and Bai (2003). In the definitions of $R_{a}$ and $R_{b}$, one subtle point is that $\Sigma_{a}$ and $\Sigma_{b}$ are averages that depend on $N$, whereas $\Sigma_{F}$ and $\Sigma_{\bar{F}}$ are asymptotic limits as $T \rightarrow \infty$. This subtle difference is crucial for deriving asymptotic limits of the PLS estimators if potential structural change is considered. In the absence of structural instabilities, $R_{a}=R_{b}$ by construction. This immediately implies that $\Gamma^{R}=0$ for any $N$, instead of $\Gamma^{R} \rightarrow 0$, as both $N$ and $T$ go to infinity. As previously stated, let the subscript $\ell$ denote the $\ell$-th column of a matrix.

Theorem 1 Suppose Assumptions A-D, P1-P2, and T hold. Then,

(a) Pre-break loadings of relevant factors: $N^{-1}\left\|\widehat{\Lambda}_{\ell}-\Lambda_{\ell}^{R}\right\|^{2}=O_{p}\left(C_{N T}^{-2}\right)$ for $\ell=1, \ldots, r_{a}$;

(b) Pre-break loadings of irrelevant factors: $\operatorname{Pr}\left(\left\|\widehat{\Lambda}_{\ell}\right\|^{2}=0\right.$ for $\left.\ell=r_{a}+1, \ldots, k\right) \rightarrow 1$;

(c) Post-break changes in loadings of relevant factors: If $\Gamma^{0} \neq 0, N^{-1}\left\|\widehat{\Gamma}_{\ell}-\Gamma_{\ell}^{R}\right\|^{2}=O_{p}\left(C_{N T}^{-2}\right)$ for $\ell=1, \ldots, r_{b}$;

(d) No-break: If $\Gamma^{0}=0, \operatorname{Pr}\left(\left\|\widehat{\Gamma}_{\ell}\right\|^{2}=0\right.$ for $\left.1, \ldots, r_{b}\right) \rightarrow 1$;

(e) Post-break changes in loadings of irrelevant factors: $\operatorname{Pr}\left(\left\|\widehat{\Gamma}_{\ell}\right\|^{2}=0\right.$ for $\left.\ell=r_{b}+1, \ldots, k\right) \rightarrow$ 1.

Parts (a) and (b) of Theorem 1 characterize the limits of the PLS estimators of the prebreak factor loadings. Due to the penalization, the factor loadings of the irrelevant factors are estimated as exactly zero w.p.a.1. This superefficiency result cannot be achieved by the unrestricted least square estimators. In contrast, for the true factors, the penalization does not affect the consistency and the convergence rate of their estimators. For $\ell=1, \ldots, r_{a}$, the PLS estimator $\widehat{\Lambda}_{\ell}$ converges in probability to the factor loadings $\Lambda_{\ell}^{R}$ of the transformed DGP. 
Parts (c) to (e) of Theorem 1 characterize asymptotic properties of the PLS estimators of the changes in the factor loadings, which is essential to detecting structural instabilities. In the absence of structural instabilities, the PLS estimators of the changes are equal to 0 w.p.a.1. In the presence of a structural instability, the superefficiency in Part (e) of Theorem 1 only applies to the redundant factors, which pins down the number of factors after the break.

To obtain results in Parts (a) and (c) of Theorem 1, only the upper bound on the convergence rate of the tuning parameters in Assumption T(i) is necessary. The lower bound in Assumption T(ii) is necessary for the superefficiency results stated in Parts (b), (d), and (e) of Theorem 1. Because the unrestricted least square estimators are special cases of the PLS estimators with zero penalties, Parts (a) and (c) of Theorem 1 apply to $\widehat{\Lambda}_{\ell}=\widetilde{\Lambda}_{\ell, L S}$ and $\widehat{\Gamma}_{\ell}=\widetilde{\Gamma}_{\ell, L S}$ for $\ell=1, \ldots, k$, regardless of the specification of the model.

\subsection{Consistent Model Selection}

In the previous subsection, we showed that the factor loadings of the irrelevant factors are estimated as zeros w.p.a.1. We also showed that the changes in the loadings of the relevant factors are estimated as zero w.p.a.1, if their loadings are not subjected to any instability. Hence, to establish the model selection consistency for the PLS estimation, it is sufficient to show that the asymptotic limits $\Lambda_{\ell}^{R}$ and $\Gamma_{\ell}^{R}$ in Parts (a) and (c) of Theorem 1 are bounded away from zero w.p.a.1.

Lemma 1 Suppose Assumptions A-D hold. Then,

(a) $N^{-1}\left\|\Lambda_{\ell}^{R}\right\|^{2}=\rho_{\ell}\left(\Sigma_{\Lambda} \Sigma_{F}\right)+o(1)$ for $\ell=1, \ldots, r_{a}$;

(b) If $r_{b}>r_{a}, N^{-1}\left\|\Gamma_{\ell}^{R}\right\|^{2}=\rho_{\ell}\left(\Sigma_{\Psi} \Sigma_{\bar{F}}\right)+o(1)$ for $\ell=r_{a}+1, \ldots, r_{b}$;

(c) If $r_{b}=r_{a}$ and $\operatorname{rank}\left(\Sigma_{\Lambda \Psi}^{+}\right)>r_{a}, N^{-1} \Gamma^{R} \Gamma^{R} \rightarrow \Sigma_{\Gamma}$ for some $\Sigma_{\Gamma} \neq 0$ as $N \rightarrow \infty$;

(d) $N^{-1}\left\|\Gamma_{\ell}^{R}\right\|^{2} \geq\left[\sqrt{\rho_{\ell}\left(\Sigma_{\Psi} \Sigma_{\bar{F}}\right)}-\sqrt{\rho_{\ell}\left(\Sigma_{\Lambda} \Sigma_{F}\right)}\right]^{2}+o(1)$ for $\ell=1, \ldots, r_{a}$.

Lemma 1(a) follows from the definition of the transformation matrix $R_{a}$ in (2.8) and, together with Theorem 1(a) and (c), implies that $\left\|\widehat{\Lambda}_{\ell}\right\| \neq 0$ for $\ell \leq r_{a}$ and $\left\|\widehat{\Lambda}_{\ell}\right\|=0$ for $\ell>r_{a}$ w.p.a.1. Therefore, a consistent estimator of $r_{a}$ is the last nonzero column in $\widehat{\Lambda}$. For the type- 2 instability with $r_{b}>r_{a}$, the same arguments apply by combining Lemma 1(b) 
with Theorem 1(c) and (e). It follows that a consistent estimator of $r_{b}$ is the last nonzero column in $\widehat{\Gamma}$.

To consistently detect a type- 1 instability (i.e., $r_{a}=r_{b}$ and $\Gamma_{1}^{0} \neq 0$ ), it is sufficient to show that $N^{-1}\left\|\Gamma^{R}\right\|^{2}$ is bounded away from 0 asymptotically. Lemma 1(c) focuses on the change of the space spanned by the factor loadings. One sufficient condition for Lemma 1(c) is Assumption ID(i), which holds if and only if $\Lambda^{0}$ and $\Psi^{0}$ do not span the same column space asymptotically. Lemma 1(d) suggests that an alternative sufficient condition for identification of the structural instability is Assumption ID(ii), which focuses on the change in the scaling of factor loadings.

Theorem 2 Suppose Assumptions A-D, ID, P1, P2, and T hold. Then the model selection is consistent:

$$
\operatorname{Pr}\left(\widehat{\mathcal{M}}=\mathcal{M}_{0}\right) \rightarrow 1 \text { as } N, T \rightarrow \infty
$$

Theorem 2 provides asymptotic consistency for any set of preliminary estimators that satisfy Assumption P1 and P2. If the unrestricted least squares estimators are used as preliminary estimators, our model selection procedure is consistent under a set of primitive conditions that do not involve Assumptions P1 and P2.

Corollary 1 If $(\widetilde{\Lambda}, \widetilde{\Gamma})=\left(\widetilde{\Lambda}_{L S}, \widetilde{\Gamma}_{L S}\right)$, then Theorem 2 holds under Assumptions A-D, ID, and $\mathrm{T}$.

If the preliminary estimator is a shrinkage estimator based on a rough choice of the tuning parameters, then Theorems 1 and 2 can be applied repeatedly to a multistep shrinkage estimation procedure, which in each step fine-tunes the penalty terms based on the estimation results of the previous step. Such a multistep estimation procedure is discussed in detail in Section 5.

\section{Practical Guidance for Implementation}

We provide a practical procedure for choosing the tuning parameters $\alpha_{N T}$ and $\beta_{N T}$ in Section 5.1. Moreover, in Section 5.2, we propose a two-step shrinkage estimation procedure that fine-tunes the penalties in the second-stage based on the first-stage shrinkage estimation results. This procedure improves the finite-sample performance of the PLS estimator, and we show in Section 5.3 that it also leads to consistent model selection. 


\subsection{Choosing the Penalty Weights $\alpha_{N T}$ and $\beta_{N T}$}

According to Theorem 2, consistent model selection requires $\alpha_{N T}$ and $\beta_{N T}$ to converge to 0 at least as fast as $N^{-1 / 2} C_{N T}^{-1}$ and slower than $N^{-1 / 2} C_{N T}^{-(2 d+1)}$. In practice, we choose $\alpha_{N T}$ and $\beta_{N T}$ to balance these two rates. For two scaling constants $\kappa_{1}$ and $\kappa_{2}$, let

$$
\alpha_{N T}=\kappa_{1} N^{-1 / 2} C_{N T_{a}}^{-d-1} \text { and } \beta_{N T}=\kappa_{2} N^{-1 / 2} C_{N T_{b}}^{-d-1},
$$

where $C_{N T_{a}}=\min \left(N^{1 / 2}, T_{a}^{1 / 2}\right)$ and $C_{N T_{b}}=\min \left(N^{1 / 2}, T_{b}^{1 / 2}\right)$. Although $C_{N T_{a}}, C_{N T_{b}}$, and $C_{N T}$ are all of the same asymptotic order, we use $C_{N T_{a}}$ and $C_{N T_{b}}$ rather than $C_{N T}$ in (5.1) to improve the finite-sample accuracy. Roughly speaking, $\alpha_{N T}$ is the weight attached to the penalty on the coefficients related to $X_{a}$, whereas $\beta_{N T}$ is the penalty weight on the coefficients of $X_{b}$.

In choosing the scaling constants $\kappa_{1}$ and $\kappa_{2}$, we consider the optimality conditions that lead the PLS estimators to have zero solutions for some columns in $\Lambda$ and/or $\Gamma$. Intuitively, the criterion function in (3.2) is minimized at 0 if the marginal penalty for deviating from 0 is larger than the marginal gain on the least square criterion function. Translated into our notation, $\left\|\widehat{\Lambda}_{\ell}\right\|=0$ for $\ell>r_{a}$ if

$$
\left\|e_{a}(\widehat{\Lambda}) \widetilde{F}_{a, \ell}+e_{b}(\widehat{\Lambda}+\widehat{\Gamma}) \widetilde{F}_{b, \ell}\right\|<N T \alpha_{N T} \omega_{\ell}^{\lambda} / 2,
$$

where the residual matrices are

$$
e_{a}(\Lambda)=X_{a}-\widetilde{F}_{a} \Lambda^{\prime} \text { and } e_{b}(\Lambda+\Gamma)=X_{b}-\widetilde{F}_{b}(\Lambda+\Gamma)^{\prime} .
$$

The inequality in (5.2) suggests that doubling every element in the residual matrices $e_{a}(\Lambda)$ and $e_{b}(\Lambda+\Gamma)$ has to be compensated for by doubling $\kappa_{1}$ to ensure that the inequality in (5.2) holds. Therefore, to standardize the left-hand side of (5.2), a reasonable choice of $\kappa_{1}$ is

$$
\kappa_{1}=\frac{1}{\zeta}\left\{\left(N T_{a}\right)^{-1 / 2}\left\|e_{a}(\widetilde{\Lambda})\right\|+\left(N T_{b}\right)^{-1 / 2}\left\|e_{b}(\widetilde{\Lambda}+\widetilde{\Gamma})\right\|\right\}
$$

where $\zeta=1$ by default.

In practice, we also consider $\zeta=2,4$, and 6 to check the sensitivity of the estimation results to the penalty function. Although our asymptotic theory does not apply to the weak factors considered in Onatski (2012), the adjustment of $\zeta$ corresponds to the strength of the factors in finite samples. Generally speaking, a larger $\zeta$ works better on detecting a weak factor and a small break, while a smaller $\zeta$ works better for strong factors and large breaks. 
If the break occurs at the end of the sample, simulation results suggest that a larger $\zeta$, e.g., $\zeta=4$, tends to work better than $\zeta=1$. By similar arguments, the choice of $\kappa_{2}$ is

$$
\kappa_{2}=\frac{1}{\zeta}\left(N T_{b}\right)^{-1 / 2}\left\|e_{b}(\widetilde{\Lambda}+\widetilde{\Gamma})\right\|
$$

In sum, the recommended tuning parameters are:

$$
\begin{aligned}
\alpha_{N T} & =\frac{1}{\zeta}\left(\left(N T_{a}\right)^{-1 / 2}\left\|e_{a}(\widetilde{\Lambda})\right\|+\left(N T_{b}\right)^{-1 / 2}\left\|e_{b}(\widetilde{\Lambda}+\widetilde{\Gamma})\right\|\right) N^{-1 / 2} C_{N T_{a}}^{-d-1}, \\
\beta_{N T} & =\frac{1}{\zeta}\left(\left(N T_{b}\right)^{-1 / 2}\left\|e_{b}(\widetilde{\Lambda}+\widetilde{\Gamma})\right\|\right) N^{-1 / 2} C_{N T_{b}}^{-d-1} .
\end{aligned}
$$

\subsection{Two-Step Estimation Procedure}

We recommend a two-step estimation procedure that uses as preliminary estimators in the second step the shrinkage estimators from the first step. The two-step procedure improves the finite sample performance through two channels. First, the tuning parameters are better calibrated in the second step because the residual matrices in (5.6) are more accurate when $\widetilde{\Lambda}$ and $\widetilde{\Gamma}$ are based on first-step shrinkage estimators. Second, a better preliminary estimator $\widetilde{\Gamma}$ is obtained through a rotation of the factor loadings $\Lambda^{R}$ and $\Psi^{R}$. For $i=1$ and 2 , let $\widetilde{\Lambda}^{(i)}, \widetilde{\Psi}^{(i)}$, and $\widetilde{\Gamma}^{(i)}$ denote the preliminary estimators, $\widehat{\Lambda}^{(i)}, \widehat{\Psi}^{(i)}$ and $\widehat{\Gamma}^{(i)}$ denote the PLS

estimators, and $\widehat{\Lambda}_{P M S}^{(i)}, \widehat{\Psi}_{P M S}^{(i)}$ and $\widehat{\Gamma}_{P M S}^{(i)}$ denote the PMS estimators in step $i$. The two-step estimation can be implemented with the following algorithm:

\section{Algorithm 1 (Two-Step Estimation Procedure) Execute the following steps:}

1. Construct the first-stage shrinkage estimator as follows:

(a) Compute the unrestricted least squares estimators $\widetilde{\Lambda}_{L S}$ and $\widetilde{\Lambda}_{L S}$.

(b) Let $\widetilde{\Lambda}^{(1)}=\widetilde{\Lambda}_{L S}$ and $\widetilde{\Gamma}^{(1)}=\widetilde{\Gamma}_{L S}$. Calculate $\omega_{\ell}^{\lambda}, \omega_{\ell}^{\gamma}, \alpha_{N T}$ and $\beta_{N T}$ following (3.4) and (5.6) with $\widetilde{\Lambda}=\widetilde{\Lambda}^{(1)}$ and $\widetilde{\Gamma}=\widetilde{\Gamma}^{(1)}$.

(c) Compute the shrinkage estimator $\widehat{\Lambda}^{(1)}$ and $\widehat{\Gamma}^{(1)}$ by minimizing the criterion function (3.2).

(d) Estimate $r_{a}$ and $r_{b}$ following (3.5) with $\widehat{\Lambda}=\widehat{\Lambda}^{(1)}$ and $\widehat{\Gamma}=\widehat{\Lambda}^{(1)}$ and call the estimators $\widehat{r}_{a}^{(1)}$ and $\widehat{r}_{b}^{(1)}$. Let $\bar{\Lambda}^{(1)}$ denote the first $\widehat{r}_{a}^{(1)}$ columns of $\widetilde{\Lambda}_{L S}$ and $\bar{\Psi}^{(1)}$ 
denote the first $\widehat{r}_{b}^{(1)}$ columns of $\widetilde{\Psi}_{L S}$. Following the definition of the PMS estimator in (3.9),

$$
\widehat{\Lambda}_{P M S}^{(1)}=\left(\bar{\Lambda}^{(1)}, 0_{\Lambda^{(1)}}\right) \in R^{N \times k} \text { and } \widehat{\Psi}_{P M S}^{(1)}=\left(\bar{\Psi}^{(1)}, 0_{\Psi^{(1)}}\right) \in R^{N \times k}
$$

where $0_{\Lambda^{(1)}}$ is a $N \times\left(k-\widehat{r}_{a}^{(1)}\right)$ zero matrix and $0_{\Psi^{(1)}}$ is a $N \times\left(k-\widehat{r}_{b}^{(1)}\right)$ zero matrix.

(e) If $\widehat{r}_{b}^{(1)}=\widehat{r}_{a}^{(1)}$, transform the columns of $\bar{\Psi}^{(1)}$ as follows: Let $\bar{\Lambda}^{(1)^{\prime}} \bar{\Psi}^{(1)}=U D V^{\prime}$ be the singular value decomposition of $\bar{\Lambda}^{(1)^{\prime}} \bar{\Psi}^{(1)}=U D V^{\prime}$. Define the transformed factor loading as

$$
\bar{\Psi}_{R}^{(1)}=\bar{\Psi}^{(1)} Q,
$$

where $Q=V U^{\prime}$. Define the modified PMS estimator of $\Psi$ as

$$
\widehat{\Psi}_{P M S-R}^{(1)}=\left(\bar{\Psi}_{R}^{(1)}, 0_{\Psi^{(1)}}\right) \in R^{N \times k},
$$

which is a modification of $\widehat{\Psi}_{P M S}^{(1)}$ with $\bar{\Psi}^{(1)}$ replaced by $\bar{\Psi}_{R}^{(1)}$.

2. Construct the second-stage shrinkage estimator as follows:

(a) Let

$$
\widetilde{\Lambda}^{(2)}=\widehat{\Lambda}_{P M S}^{(1)}, \quad \widetilde{\Psi}^{(2)}=\left\{\begin{array}{cl}
\widehat{\Psi}_{P M S-R}^{(1)} & \text { if } \widehat{r}_{b}^{(1)}=\widehat{r}_{a}^{(1)} \\
\widehat{\Psi}_{P M S}^{(1)} & \text { if } \widehat{r}_{b}^{(1)}>\widehat{r}_{a}^{(1)}
\end{array}, \quad \widetilde{\Gamma}^{(2)}=\widetilde{\Psi}^{(2)}-\widetilde{\Lambda}^{(2)}\right.
$$

and calculate $\omega_{\ell}^{\lambda}, \omega_{\ell}^{\gamma}, \alpha_{N T}$, and $\beta_{N T}$ following (3.4) and (5.6) with $\widetilde{\Lambda}=\widetilde{\Lambda}^{(2)}$ and $\widetilde{\Gamma}=\widetilde{\Gamma}^{(2)}$.

(b) Compute the shrinkage estimators $\widehat{\Lambda}^{(2)}$ and $\widehat{\Gamma}^{(2)}$ by minimizing the criterion function (3.2).

(c) Estimate $r_{a}, r_{b}$, and $\mathcal{S}_{0}$ following (3.5)-(3.7) with $\widehat{\Lambda}=\widehat{\Lambda}^{(2)}$ and $\widehat{\Gamma}=\widehat{\Lambda}^{(2)}$.

The preliminary estimators in the second step are based on the PMS estimators of the first step. The rotation in Step 1(e) minimizes the risk of falsely reporting a structural break when there is no instability in the data. It is designed to match the column spaces of $\bar{\Lambda}^{(1)}$ and $\bar{\Psi}^{(1)}$. This leads to a smaller $\widetilde{\Gamma}$ if $\Gamma^{0}=0$. Formally, the problem is to find an orthogonal matrix $Q$ such that $\left\|\bar{\Lambda}^{(1)}-\bar{\Psi}^{(1)} Q\right\|_{2}$ is minimized. This is an orthogonal procrustes problem. It is equivalent to maximizing the correlation between the columns of $\bar{\Lambda}^{(1)}$ and $\bar{\Psi}^{(1)} Q$ (see 
Section 2.3). The solution is $Q=V U^{\prime}$ (see Schönemann (1966)), where $U$ and $V$ are obtained from the singular value decomposition $\bar{\Lambda}^{(1)^{\prime}} \bar{\Psi}^{(1)}=U D V^{\prime}$.

In the remainder of this subsection, we show that if there is indeed a type-1 instability, the $Q$ rotation will not eliminate the difference between $\Lambda_{\ell}^{R}$ and $\Psi_{\ell}^{R}$. Moreover, we show that the asymptotic theory we established in the previous section applies to the two-step shrinkage estimator.

\subsection{Large Sample Behavior of Two-Step Estimation Procedure}

Under Assumption ID, Lemma 1(c) and Lemma 1(d) imply that, in the presence of a type-1 change, there exists a set of columns such that

$$
\mathcal{Z}=\left\{\ell: N^{-1}\left\|\Gamma_{\ell}^{R}\right\|^{2}=N^{-1}\left\|\Psi_{\ell}^{R}-\Lambda_{\ell}^{R}\right\|^{2} \geq C\right\}
$$

The columns in the set $\mathcal{Z}$ are crucial for the identification of a type- 1 instability. We now state the following additional assumption:

Assumption R. If $r_{a}=r_{b}$, then $\inf _{\|\mathrm{w}\|=1} N^{-1}\left\|\Psi^{R} \mathrm{w}-\Lambda_{\ell}^{R}\right\|^{2} \geq C$ for $\ell \in \mathcal{Z}$.

Assumption $\mathrm{R}$ is not restrictive. It holds whenever $\Lambda_{\ell}^{R}$ is not in the column space generated by $\Psi^{R}$. Assumption $\mathrm{R}$ is imposed on the loadings $\Lambda^{R}$ of the normalized version of the DGP rather than on the loadings $\Lambda^{0}$ of the DGP itself. Assumption $\mathrm{R}$ allows the loadings of some of the "structural" factors in the unnormalized DGP to remain constant while the loadings of other "structural" factors change. In the absence of structural instabilities, $\mathcal{Z}$ is empty and Assumption $\mathrm{R}$ is not necessary.

Using Assumption R, the model selection consistency established in Theorem 2 can be extended to the two-step estimation procedure, as summarized in the following corollary:

Corollary 2 If $\widetilde{\Lambda}=\widetilde{\Lambda}^{(2)}$ and $\widetilde{\Gamma}=\widetilde{\Gamma}^{(2)}$, then Theorem 2 holds under Assumptions A-D, ID, $\mathrm{R}$, and $\mathrm{T}$.

Recall that Assumptions A-D, ID, and R are primitive conditions on the factor models. The recommended tuning parameters $\alpha_{N T}$ and $\beta_{N T}$ defined in (5.6) are constructed to ensure that Assumption $\mathrm{T}$ is satisfied. As a result, one can continue to use the recommended formulas of $\alpha_{N T}$ and $\beta_{N T}$ throughout the two-step estimation procedure. 


\section{Model Selection with Unknown Break Date}

We now extend the model selection procedure to study a potential one-time abrupt break at an unknown time $T_{0}$, allowing for both type- 1 and type- 2 instabilities. Let $\pi_{0}=T_{0} / T$, where $T$ is the number of periods in the sample. For simplicity, we call $\pi_{0}$, rather than $T_{0}$, the break date. We assume that the exact value of $\pi_{0}$ is unknown, but it is known that $\pi_{0} \in \Pi$, where $\Pi$ is some closed subset in the interior of $[0,1]$.

For any $\pi \in \Pi$, we split the full sample into two subsamples $X_{a}(\pi)=\left(X_{1}, \ldots, X_{T_{a}}\right)^{\prime} \in$ $R^{T_{a} \times N}$ and $X_{b}(\pi)=\left(X_{T_{a}+1}, \ldots, X_{T}\right)^{\prime} \in R^{T_{b} \times N}$, where $T_{a}=\lfloor T \pi\rfloor$ is the integer part of $T \pi$ and $T_{b}=T-T_{a}$. In Section 6.1, we study the consequences of misspecifying the break date and present an identification condition for the unknown break date. To account for the unknown break date, we slightly modify the penalty terms of the PLS estimator in Section 6.2. Finally, we show in Section 6.3 that the consistency result of Theorem 2 extends to the case with an unknown break date.

\subsection{Identification of the Change Point}

To obtain an identification condition for the unknown break date $\pi_{0}$, we now study the number of factors in $X_{a}(\pi)$ and $X_{b}(\pi)$ when $\pi \neq \pi_{0}$. In a nutshell, if the break date is misspecified, then one of the two subsamples contains one or more additional factors. Thus, the break date can be identified by minimizing the number of estimated pre- and post-break factors by varying $\pi$.

Consider the case of $\pi<\pi_{0}$. For the first subsample $X_{a}(\pi)$, the DGP is the same as that in (2.2), which can be written as

$$
\begin{aligned}
X_{a}(\pi) & =F_{a}(\pi) \Lambda^{0 \prime}+e_{a}(\pi), \text { where } \\
F_{a}(\pi) & =\left(F_{1}^{0}, \ldots, F_{T_{a}}^{0}\right)^{\prime} \in R^{T_{a} \times r_{a}}, \\
e_{a}(\pi) & =\left(e_{1}, \ldots, e_{T_{a}}\right)^{\prime} \in R^{T_{a} \times N} .
\end{aligned}
$$

For the second subsample $X_{b}(\pi)$, which includes observations for $t=T_{a}+1, \ldots, T_{0}, \ldots, T$, the DGP corresponds to (2.2) for $t \leq T_{0}$ and to (2.3) for $t>T_{0}$. Thus, the DGP for $X_{b}(\pi)$ 
can be written as

$$
\begin{aligned}
X_{b}(\pi) & =F_{a}^{+}(\pi) \Lambda^{0 \prime}+F_{b}(\pi) \Psi^{0 \prime}+e_{b}(\pi), \text { where } \\
F_{a}^{+}(\pi) & =\left(F_{T_{a}+1}^{0}, \ldots, F_{T_{0}}^{0}, 0_{r_{a} \times\left(T-T_{0}\right)}\right)^{\prime} \in R^{T_{b} \times r_{a}}, \\
F_{b}(\pi) & =\left(0_{r_{b} \times\left(T_{0}-T_{a}\right)}, \bar{F}_{T_{0}+1}^{0}, \ldots, \bar{F}_{T}^{0}\right)^{\prime} \in R^{T_{b} \times r_{b}}, \\
e_{b}(\pi) & =\left(e_{T_{a}+1}, \ldots, e_{T}\right)^{\prime} \in R^{T_{b} \times N} .
\end{aligned}
$$

Here the $r_{a}$ factors in $F_{a}^{+}(\pi)$ with loadings $\Lambda^{0}$ are only for observations before the true break date, and the $r_{b}$ factors in $F_{b}(\pi)$ with loadings $\Psi^{0}$ are only for observations after the true break. By construction, $F_{a}^{+}(\pi)$ and $F_{b}(\pi)$ are orthogonal to each other. By definition, $F_{a}\left(\pi_{0}\right)=F_{a}, F_{a}^{+}\left(\pi_{0}\right)=0$, and $F_{b}\left(\pi_{0}\right)=F_{b}$. The DGPs in (6.1) and (6.2) reduce to (2.2) and (2.3), respectively, if the break date is known.

We now replace Assumptions $\mathrm{A}$ and $\mathrm{C}$ with Assumptions $\mathrm{A}^{*}$ and $\mathrm{C}^{*}$ such that the weak dependence and stationarity hold for any subsamples considered.

Assumption $\mathbf{A}^{*}$. $\mathbb{E}\left\|F_{t}^{0}\right\|^{4} \leq C, \mathbb{E}\left\|\bar{F}_{t}^{0}\right\|^{4} \leq C$ and there exist some positive definite matrices $\Sigma_{F}$ and $\Sigma_{\bar{F}}$ such that $T^{-1} \sum_{t=1}^{\lfloor T \pi} F_{t}^{0} F_{t}^{0 \prime}=\pi \Sigma_{F}+O_{p}\left(T^{-1 / 2}\right)$ for $\pi \leq \pi_{0}$ and $T^{-1} \sum_{t=\lfloor T \pi\rfloor+1}^{T} \bar{F}_{t}^{0} \bar{F}_{t}^{0 \prime}=(1-\pi) \Sigma_{\bar{F}}+O_{p}\left(T^{-1 / 2}\right)$ for $\pi \geq \pi_{0}$, where both $O_{p}\left(T^{-1 / 2}\right)$ terms are uniform over $\pi \in \Pi$.

Assumption $\mathrm{C}^{*}$. Assumption $\mathrm{C}$ holds with $e_{a}$ and $e_{b}$ replaced by $e_{a}(\pi)$ and $e_{b}(\pi)$ and Assumption $\mathrm{C}(\mathrm{vi})$ holds uniformly over $\pi \in \Pi$.

Let $r_{a}(\pi)$ and $r_{b}(\pi)$ denote the number of factors in $X_{a}(\pi)$ and $X_{b}(\pi)$, respectively. By definition, they are the number of nonvanishing eigenvalues of $(N T)^{-1} X_{a}(\pi)^{\prime} X_{a}(\pi)$ and $(N T)^{-1} X_{b}(\pi)^{\prime} X_{b}(\pi)$, respectively, as $N, T \rightarrow \infty$. Under Assumptions $\mathrm{A}^{*}, \mathrm{~B}, \mathrm{C}^{*}$, and $\mathrm{D}$, the DGP in (6.1) is a factor model with $r_{a}$ factors (i.e., $r_{a}(\pi)=r_{a}$ for $\pi \leq \pi_{0}$ ). To study the number of factors in (6.2) for the second subsample, note that

$$
\begin{aligned}
& T^{-1}\left(F_{a}^{+}(\pi), F_{b}(\pi)\right)^{\prime}\left(F_{a}^{+}(\pi), F_{b}(\pi)\right) \rightarrow_{p} \Sigma_{F}^{+}(\pi), \\
& N^{-1}\left(\Lambda^{0}, \Psi^{0}\right)^{\prime}\left(\Lambda^{0}, \Psi^{0}\right) \rightarrow_{p} \Sigma_{\Lambda \Psi}^{+}, \text {where } \\
& \Sigma_{F}^{+}(\pi)=\left[\begin{array}{cc}
\left(\pi_{0}-\pi\right) \Sigma_{F} & 0_{r_{a} \times r_{b}} \\
0_{r_{b} \times r_{a}} & \left(1-\pi_{0}\right) \Sigma_{\bar{F}}
\end{array}\right]
\end{aligned}
$$

and $\Sigma_{\Lambda \Psi}^{+}$is defined in (2.6). Because $\Sigma_{F}^{+}(\pi)$ has full rank by construction, the number of factors in $X_{b}(\pi)$ depends on the rank of $\Sigma_{\Lambda \Psi}^{+}$(i.e., $r_{b}(\pi)=\operatorname{rank}\left(\Sigma_{\Lambda \Psi}^{+}\right)$for $\left.\pi<\pi_{0}\right)$. If $\Lambda^{0}=\Psi^{0}$, we know that $\operatorname{rank}\left(\Sigma_{\Lambda \Psi}^{+}\right)=r_{b}$. If, on the other hand, the column spaces generated 
by $\Psi^{0}$ and $\Lambda^{0}$ do not overlap, we have $\operatorname{rank}\left(\Sigma_{\Lambda \Psi}^{+}\right)=r_{a}+r_{b}$. Typically, we would expect the column spaces generated by $\Psi^{0}$ and $\Lambda^{0}$ to be overlapping but non-nested, which means $\operatorname{rank}\left(\Sigma_{\Lambda \Psi}^{+}\right)>r_{b} \geq r_{a}$ and hence $r_{b}(\pi)>r_{b}$ for $\pi<\pi_{0}$.

Now consider $\pi>\pi_{0}$. For the first subsample, we have $r_{a}(\pi)=\operatorname{rank}\left(\Sigma_{\Lambda \Psi}^{+}\right)$, which implies $r_{a}(\pi) \geq r_{b} \geq r_{a}$, while for the second subsample, we simply get $r_{b}(\pi)=r_{b}$. The following lemma provides a summary:

Lemma 2 Suppose that Assumptions $\mathrm{A}^{*}, \mathrm{~B}, \mathrm{C}^{*}$, and $\mathrm{D}$ hold. Then,

$$
r_{a}(\pi)=\left\{\begin{array}{cc}
r_{a} & \pi \leq \pi_{0} \\
\operatorname{rank}\left(\Sigma_{\Lambda \Psi}^{+}\right) & \pi>\pi_{0}
\end{array} \quad \text { and } r_{b}(\pi)=\left\{\begin{array}{cc}
\operatorname{rank}\left(\Sigma_{\Lambda \Psi}^{+}\right) & \pi<\pi_{0} \\
r_{b} & \pi \geq \pi_{0}
\end{array}\right.\right.
$$

where $\operatorname{rank}\left(\Sigma_{\Lambda \Psi}^{+}\right) \geq r_{b} \geq r_{a}$.

It follows from Lemma 2 that

$$
r_{a}(\pi)+r_{b}(\pi)=\left\{\begin{array}{cl}
r_{a}+\operatorname{rank}\left(\Sigma_{\Lambda \Psi}^{+}\right) & \pi<\pi_{0} \\
r_{a}+r_{b} & \pi=\pi_{0} \\
r_{b}+\operatorname{rank}\left(\Sigma_{\Lambda \Psi}^{+}\right) & \pi>\pi_{0}
\end{array}\right.
$$

Because $\operatorname{rank}\left(\Sigma_{\Lambda \Psi}^{+}\right) \geq r_{b} \geq r_{a}$, we see that $r_{a}(\pi)+r_{b}(\pi)$ is minimized at $\pi_{0}$, with the minimum value $r_{a}+r_{b}$. Define the set of values $\pi$ such that $r_{a}(\pi)+r_{b}(\pi)$ achieve the smallest value $r_{a}+r_{b}$ as

$$
\mathcal{D}=\left\{\pi \in \Pi: r_{a}(\pi)+r_{b}(\pi)=r_{a}+r_{b}\right\}
$$

By definition, we know that $\pi_{0} \in \mathcal{D}$ and hence $\mathcal{D}$ is a well-defined nonempty set. If $\operatorname{rank}\left(\Sigma_{\Lambda \Psi}^{+}\right)>r_{b}$ (which holds if the column space generated by $\Lambda^{0}$ is not contained by that generated by $\Psi^{0}$ asymptotically), we can deduce that $\pi_{0}$ is the unique minimizer. The result is summarized in the following corollary:

Corollary 3 Suppose that Assumptions $\mathrm{A}^{*}, \mathrm{~B}, \mathrm{C}^{*}$, and $\mathrm{D}$ hold.

(a) If $\operatorname{rank}\left(\Sigma_{\Lambda \Psi}^{+}\right)>r_{b}$, then $\pi_{0}=\mathcal{D}$;

(b) If $\operatorname{rank}\left(\Sigma_{\Lambda \Psi}^{+}\right)=r_{b}>r_{a}$, then $\pi_{0}=\max \{\pi: \pi \in \mathcal{D}\}$. 
If the structural break is due to the type- 2 instability, Corollary 3 shows that the break date $\pi_{0}$ can always be identified by the maximum value in the set $\mathcal{D}$. $\pi_{0}$ can still be identified under type-1 instability, provided that Assumption ID(i) holds. In that case, we have

$$
\operatorname{rank}\left(\Sigma_{\Lambda \Psi}^{+}\right)>r_{a}=r_{b}
$$

which combined with Corollary $3\left(\right.$ a) implies that $\left\{\pi_{0}\right\}=\mathcal{D}$. The only case in which $\mathcal{D}$ does not identify $\pi_{0}$ is if $r_{a}=r_{b}$ and $\Lambda^{0}$ and $\Psi^{0}$ span the same column spaces. However, this type-1 instability itself can still be identified as long as Assumption ID(ii) holds.

\subsection{Estimation with Unknown Break Date}

For any $\pi \in \Pi$, let $\widetilde{F}_{a}(\pi) \in R^{T_{a} \times k}$ be the orthonormalized eigenvectors of $\left(N T_{a}\right)^{-1} X_{a}(\pi) X_{a}(\pi)^{\prime}$ associated with its first $k$ largest eigenvalues. Similarly, let $\widetilde{F}_{b} \in R^{T_{b} \times k}$ be the orthonormalized left eigenvectors of $\left(N T_{b}\right)^{-1} X_{b}(\pi) X_{b}(\pi)^{\prime}$ associated with its first $k$ largest eigenvalues. We assume $k \geq r_{a}+r_{b}$, the largest possible number of factors in any subsample. The unrestricted estimators of the factor loadings are $\widetilde{\Lambda}_{L S}(\pi)=T_{a}^{-1} X_{a}(\pi)^{\prime} \widetilde{F}_{a}(\pi)$, $\widetilde{\Psi}_{L S}(\pi)=T_{b}^{-1} X_{b}(\pi)^{\prime} \widetilde{F}_{b}(\pi)$, and $\widetilde{\Gamma}_{L S}(\pi)=\widetilde{\Psi}_{L S}(\pi)-\widetilde{\Lambda}_{L S}(\pi)$.

By applying the procedure in Section 5 with $\pi_{0}$ replaced by $\pi$, we obtain a shrinkage estimator indexed by $\pi \in \Pi$, which yields consistent estimators of $r_{a}(\pi)$ and $r_{b}(\pi)$ for any $\pi \in \Pi$. In our empirical application, we found that this simple procedure is undesirable because the estimators of $r_{a}(\pi)$ and $r_{b}(\pi)$ are highly sensitive to $\pi$. To improve the finite sample performance of the PLS estimation with an unknown break date, we propose an averaging penalty. Based on this averaging penalty, the shrinkage estimator depends on $\pi$ only through the least square criterion function. Uniform convergence of the least square criterion function over $\pi \in \Pi$ follows from Assumptions $\mathrm{A}^{*}$ and $\mathrm{C}^{*}$. In the remainder of this subsection, we describe the construction of the averaging penalty.

The shrinkage estimator with an averaging penalty is obtained by minimizing a PLS criterion function indexed by $\pi$ :

$$
(\widehat{\Lambda}(\pi), \widehat{\Gamma}(\pi))=\underset{\Lambda \in R^{N \times k}, \Gamma \in R^{N \times k}}{\arg \min }\left[M(\Lambda, \Gamma ; \pi)+P_{1}^{*}(\Lambda)+P_{2}^{*}(\Gamma)\right]
$$

where

$$
M(\Lambda, \Gamma ; \pi)=(N T)^{-1}\left[\left\|X_{a}(\pi)-\widetilde{F}_{a}(\pi) \Lambda^{\prime}\right\|^{2}+\left\|X_{b}(\pi)-\widetilde{F}_{b}(\pi)(\Lambda+\Gamma)^{\prime}\right\|^{2}\right]
$$


The averaging penalty functions $P_{1}^{*}(\Lambda)$ and $P_{2}^{*}(\Lambda)$ are

$$
\begin{aligned}
& P_{1}^{*}(\Lambda)=\sum_{\ell=1}^{k} \mathbb{E}_{\xi}\left[\alpha_{N T}(\xi) \omega_{\ell}^{\lambda *}(\xi)\right]\left\|\Lambda_{\ell}\right\|, \\
& P_{2}^{*}(\Gamma)=\sum_{\ell=1}^{k} \mathbb{E}_{\xi}\left[\beta_{N T}(\xi) \omega_{\ell}^{\gamma *}(\xi)\right]\left\|\Gamma_{\ell}\right\|,
\end{aligned}
$$

where $\xi$ has a uniform distribution on $\Pi$ and $\mathbb{E}_{\xi}[\cdot]$ denotes the expectation with respect to $\xi .^{5}$ In practice, $\Pi$ is approximated by a set of equally spaced grid points $\Pi_{d}$, and the expectation in (6.10) is replaced by an average. The tuning parameters $\alpha_{N T}(\pi)$ and $\beta_{N T}(\pi)$ are

$$
\alpha_{N T}(\pi)=\kappa_{1}(\pi) N^{-1 / 2} C_{N T_{a}}^{-d-1} \text { and } \beta_{N T}(\pi)=\kappa_{2}(\pi) N^{-1 / 2} C_{N T_{b}}^{-d-1}
$$

where $\kappa_{1}(\pi) \in\left[\underline{\kappa}_{1}, \bar{\kappa}_{1}\right]$ and $\kappa_{2}(\pi) \in\left[\underline{\kappa}_{2}, \bar{\kappa}_{2}\right]$ for some $\underline{\kappa}_{1}, \underline{\kappa}_{2}>0$ and $\bar{\kappa}_{1}, \bar{\kappa}_{2}<\infty$. They are analogous to those in (5.1). In practice, we can choose $\kappa_{1}(\pi)$ and $\kappa_{2}(\pi)$ as in (5.6) but with $\widetilde{\Lambda}$ and $\widetilde{\Gamma}$ replaced by $\widetilde{\Lambda}(\pi)$ and $\widetilde{\Gamma}(\pi)$, respectively.

For each $\pi \in \Pi$, let $\widetilde{\Lambda}(\pi), \widetilde{\Psi}(\pi)$, and $\widetilde{\Gamma}(\pi)$ be some preliminary estimators. We define adaptive weights $\omega_{\ell}^{\lambda *}(\pi)$ and $\omega_{\ell}^{\gamma *}(\pi)$ as

$$
\begin{aligned}
\omega_{\ell}^{\lambda *}(\pi)= & \left(N^{-1}\left\|\widetilde{\Lambda}_{\ell}(\pi)\right\|^{2} \mathcal{I}_{\left\{\widetilde{\Lambda}_{\ell}(\pi) \neq 0_{N \times 1}\right\}}+N^{-1}\left\|\widetilde{\Lambda}_{\ell, L S}(\pi)\right\|^{2} \mathcal{I}_{\left\{\widetilde{\Lambda}_{\ell}(\pi)=0_{N \times 1}\right\}}\right)^{-d}, \\
\omega_{\ell}^{\gamma *}(\pi)= & \left(N^{-1} \min \left\{\left\|\widetilde{\Gamma}_{\ell}(\pi)\right\|^{2},\left\|\widetilde{\Psi}_{\ell}(\pi)\right\|^{2}\right\} \mathcal{I}_{\left\{\widetilde{\Gamma}_{\ell}(\pi) \neq 0_{N \times 1}\right\}}\right)^{-d} \\
& +\left(N^{-1} \min \left\{\left\|\widetilde{\Gamma}_{\ell, L S}(\pi)\right\|^{2},\left\|\widetilde{\Psi}_{\ell, L S}(\pi)\right\|^{2}\right\} \mathcal{I}_{\left\{\widetilde{\Gamma}_{\ell}(\pi)=0_{N \times 1}\right\}}\right)^{-d} .
\end{aligned}
$$

Comparing the weights in (6.12) with those in (3.4), we see that $\omega_{\ell}^{\lambda *}\left(\pi_{0}\right)=\omega_{\ell}^{\lambda}$ but $\omega_{\ell}^{\gamma *}\left(\pi_{0}\right) \neq$ $\omega_{\ell}^{\gamma}$. If the break date is unknown, it is crucial to use $\omega_{\ell}^{\gamma *}(\pi)$ for consistent estimation of $r_{b}$ because, for $\pi>\pi_{0}$ and $\ell>r_{b}, N^{-1}\left\|\widetilde{\Psi}_{\ell, L S}(\pi)\right\|^{2}$ converges (in probability) to 0 , but $N^{-1}\left\|\widetilde{\Gamma}_{\ell, L S}(\pi)\right\|^{2}$ may not converge (in probability) to 0 . Thus, the modified adaptive weights can deliver larger penalties, when needed. The modified weights can also be used if the break date is known, because $N^{-1}|| \widetilde{\Gamma}_{\ell}\left(\pi_{0}\right) \|^{2}$ and $N^{-1}|| \widetilde{\Psi}_{\ell}\left(\pi_{0}\right) \|^{2}$ are either of the same order of magnitude or the former is smaller than the latter.

\footnotetext{
${ }^{5}$ By definition,

$$
\mathbb{E}_{\xi}\left[\alpha_{N T}(\xi) \omega_{\ell}^{\lambda}(\xi)\right]=\int_{\underline{\pi}}^{\bar{\pi}} \alpha_{N T}(\xi) \omega_{\ell}^{\lambda}(\xi) \frac{1}{\bar{\pi}-\underline{\pi}} d \xi \text { and } \mathbb{E}_{\xi}\left[\beta_{N T}(\xi) \omega_{\ell}^{\gamma}(\xi)\right]=\int_{\underline{\pi}}^{\bar{\pi}} \beta_{N T}(\xi) \omega_{\ell}^{\gamma}(\xi) \frac{1}{\bar{\pi}-\underline{\pi}} d \xi,
$$
}

where $\underline{\pi}$ and $\bar{\pi}$ are the lower and upper bounds of $\Pi$. Note that the above two terms depend on $N$ and $T$. 


\subsection{Model Selection with Unknown Break Date}

If the break date is unknown, the model selection for $\mathcal{M}_{0}$ is based on the zero and nonzero columns in $\widehat{\Lambda}(\pi)$ and $\widehat{\Gamma}(\pi)$ for all $\pi \in \Pi$. First, we generalize the estimators $\widehat{r}_{a}$ and $\widehat{r}_{b}$ in (3.5) to

$$
\begin{aligned}
& \widehat{r}_{a}(\pi)=\min \mathcal{J}_{a}(\pi), \text { where } \mathcal{J}_{a}(\pi)=\left\{j:\left\|\widehat{\Lambda}_{\ell}(\pi)\right\|^{2}=0 \text { for all } \ell>j\right\} \\
& \widehat{r}_{b}(\pi)=\max \left(\min \mathcal{J}_{b}, \widehat{r}_{a}(\pi)\right), \text { where } \mathcal{J}_{b}(\pi)=\left\{j:\left\|\widehat{\Gamma}_{\ell}(\pi)\right\|^{2}=0 \text { for all } \ell>j\right\} .
\end{aligned}
$$

With an unknown break date, the estimators of $r_{a}$ and $r_{b}$ are

$$
\widehat{r}_{a}^{*}=\min _{\pi \in \Pi} \widehat{r}_{a}(\pi) \text { and } \widehat{r}_{b}^{*}=\min _{\pi \in \Pi} \widehat{r}_{b}(\pi)
$$

The estimator of $\mathcal{S}_{0}$ is

$$
\widehat{\mathcal{S}}^{*}=\left\{\begin{array}{cc}
0 & \text { if } \sup _{\pi \in \Pi}|| \widehat{\Gamma}(\pi) \|=0 \\
1 & \text { otherwise }
\end{array} .\right.
$$

The selected model is

$$
\widehat{\mathcal{M}}^{*}=\left(\widehat{r}_{a}^{*}, \widehat{r}_{b}^{*}, \widehat{\mathcal{S}}^{*}\right)
$$

As in the known-break-date case, we consider a two-step procedure for model selection. Follow the steps in Section 5.2 by setting $\pi_{0}=\pi, \widetilde{\Lambda}^{(1)}(\pi)=\widetilde{\Lambda}_{L S}(\pi), \widetilde{\Psi}^{(1)}(\pi)=\widetilde{\Psi}_{L S}(\pi)$, and $\widetilde{\Gamma}^{(1)}(\pi)=\widetilde{\Gamma}_{L S}(\pi)$; replacing $\omega_{\ell}^{\lambda}, \omega_{\ell}^{\gamma}, \alpha_{N T}$, and $\beta_{N T}$ with $\omega_{\ell}^{\lambda *}(\pi), \omega_{\ell}^{\gamma *}(\pi), \alpha_{N T}(\pi)$, and $\beta_{N T}(\pi)$, respectively; replacing the PLS criterion (3.2) with (6.8); and replacing the estimators $\widehat{r}_{a}$ and $\widehat{r}_{b}$ in (3.5) with those in (6.14). Note that the first-step estimators $\widehat{r}_{a}^{(1)}$ and $\widehat{r}_{b}^{(1)}$ do not vary with $\pi$ following the definition in (6.14). Therefore, one should first obtain the firststep estimator $\widehat{\Lambda}^{(1)}(\pi)$ and $\widehat{\Gamma}^{(1)}(\pi)$ for each $\pi \in \Pi$ and get $\widehat{r}_{a}^{(1)}$ and $\widehat{r}_{b}^{(1)}$, and then obtain the second-step estimator $\widehat{\Lambda}^{(2)}(\pi)$ and $\widehat{\Gamma}^{(2)}(\pi)$ for each $\pi \in \Pi$. The selected model $\widehat{\mathcal{M}}^{*}$ is based on the two-step PLS estimator $\widehat{\Lambda}^{(2)}(\pi)$ and $\widehat{\Gamma}^{(2)}(\pi)$.

To show the model selection consistency for the two-step PLS estimator described in the previous subsection, we strengthen Assumption $\mathrm{R}$ to take into account the unknown break date and the averaging penalty. For any $\pi \in \Pi$, we can write the normalized system as

$$
\begin{aligned}
& X_{a}(\pi)=F_{a}^{R}(\pi) \Lambda^{R}(\pi)^{\prime}+e_{a}(\pi), \\
& X_{b}(\pi)=F_{b}^{R}(\pi) \Psi^{R}(\pi)^{\prime}+e_{b}(\pi),
\end{aligned}
$$

where $F_{a}^{R}(\pi)$ and $\Lambda^{R}(\pi)$ are $T_{a} \times r_{a}(\pi)$ and $N \times r_{a}(\pi)$ matrices, respectively, and $F_{b}^{R}(\pi)$ and $\Psi^{R}(\pi)$ are $T_{b} \times r_{b}(\pi)$ and $N \times r_{b}(\pi)$ matrices, respectively. 
Assumption R.*. (i) If $r_{a}=r_{b}$, then $\inf _{\pi \in \Pi} \inf _{\|\mathrm{w}\|=1} N^{-1}\left\|\Psi^{R}(\pi) \mathrm{w}-\Lambda_{\ell}^{R}(\pi)\right\|^{2} \geq C$ for $\ell \in \mathcal{Z}$

(ii) If $r_{b}>r_{a}$, then $\inf _{\pi>\pi_{0}} N^{-1}\left\|\Psi_{\ell}^{R}(\pi)-\Lambda_{\ell}^{R}(\pi)\right\|^{2} \geq C$ for $\ell=r_{b}$.

Assumption $\mathrm{R}^{*}(\mathrm{i})$ generalizes Assumption $\mathrm{R}$ from $\pi=\pi_{0}$ to any $\pi \in \Pi$. Assumption $\mathrm{R}^{*}$ (ii) is not necessary if the break date $\pi_{0}$ is known because $\Lambda_{\ell}^{R}\left(\pi_{0}\right)=0$ for $\ell=r_{b}>r_{a}$. Similar to Assumption $R$, these assumptions are not restrictive because $\Lambda_{\ell}^{R}(\pi)$ and $\Psi_{\ell}^{R}(\pi)$ are some specific matrices transformed from the structural factors. Assumptions $\mathrm{R}$ and $\mathrm{R}^{*}$ are compatible in applications where the loadings of some structural factors change and some do not.

Theorem 3 Suppose that Assumptions $\mathrm{A}^{*}, \mathrm{~B}, \mathrm{C}^{*}, \mathrm{D}$, ID, and $\mathrm{R}^{*}$ hold. Then the model selection with an unknown break date is consistent:

$$
\operatorname{Pr}\left(\widehat{\mathcal{M}}^{*}=\mathcal{M}_{0}\right) \rightarrow 1 \text { as } N, T \rightarrow \infty
$$

The identification result in Section 6.1 is used constructively in the proof of Theorem 3 . Among all the models indexed by $\pi$, only the one with the smallest number of factors is not over-penalized by the averaging penalty. Since the model with the true break date has the smallest number of factors by Lemma 2, the PLS estimator with the averaging penalty can consistently select the true model without knowing the break date.

The proof strategy of Theorem 3 is different from that of Theorem 2 due to the averaging penalty. Theorem 3 is proved by first showing the convergence of $\widehat{\Lambda}_{r_{a}}(\pi)$ and $\widehat{\Gamma}_{r_{a}}(\pi)$ uniformly over $\pi \in \Pi$. Provided that $\widehat{\Lambda}_{r_{a}}(\pi)$ uniformly converges to a nonzero limit for all $\pi \in \Pi$, it follows that $\operatorname{Pr}\left(\min _{\pi \in \Pi} \widehat{r}_{a}(\pi) \geq r_{a}\right) \rightarrow 1$. Because $\pi_{0} \in \Pi$ and $r_{a}\left(\pi_{0}\right)=r_{a}$ by definition, one can show that $\operatorname{Pr}\left(\widehat{r}_{a}\left(\pi_{0}\right)=r_{a}\right) \rightarrow 1$ as long as results like those in Theorem 1 hold for $\widehat{\Lambda}\left(\pi_{0}\right)$. Combining the two results above, we immediately get $\operatorname{Pr}\left(\min _{\pi \in \Pi} \widehat{r}_{a}(\pi)=r_{a}\right) \rightarrow$ 1. Similar arguments can be applied to $\widehat{\Gamma}_{r_{a}}(\pi)$ to show that $\operatorname{Pr}\left(\min _{\pi \in \Pi} \widehat{r}_{b}(\pi)=r_{b}\right) \rightarrow 1$. After showing consistency of the estimators of the number of factors, we analyze $\widehat{\Gamma}\left(\pi_{0}\right)$ for consistent detection of type- 1 instability, and show that $\operatorname{Pr}(\widehat{\Gamma}(\pi)=0) \rightarrow 1$ uniformly over $\pi \in \Pi$ when there are no structural instabilities.

The averaging penalty enables consistent estimation of $r_{a}$ and $r_{b}$ but does not yield consistent estimation of $\widehat{r}_{a}(\pi)$ and $\widehat{r}_{b}(\pi)$ for $\pi \neq \pi_{0}$. Therefore, the resulting shrinkage estimation does not simultaneously produce a consistent estimator of the break date. In 
practice, researchers tend to have a conjecture for the break date, which is denoted by $\pi_{c}$, and used to center the set $\Pi$ in the previous subsection. The shrinkage estimator can be used to verify whether a conjecture break date $\pi_{c}$ is a consistent estimator of $\pi_{0}$. If the data provide evidence against this conjecture, $\pi_{c}$ can be revised accordingly. We denote the revised break date by $\pi_{r c}$, and it is defined as follows: Let $\widehat{\mathcal{D}}$ denote the set of $\pi \in \Pi$ at which $\widehat{r}_{a}(\pi)+\widehat{r}_{b}(\pi)$ achieves its minimum, which typically is an interval in finite samples. Corollary 3 suggests that $\pi_{c}$ is a reasonable conjecture only if $\pi_{c} \in \widehat{\mathcal{D}}$. Hence, if $\pi_{c} \in \widehat{\mathcal{D}}$, we do not revise the conjectured break date and we define $\pi_{r c}=\pi_{c}$. On the other hand, if

$\pi_{c} \notin \widehat{\mathcal{D}}$, we choose $\pi_{r c} \in \widehat{\mathcal{D}}$ such that $\left|\pi_{r c}-\pi_{c}\right|=\min _{\pi \in \widehat{\mathcal{D}}}\left|\pi-\pi_{c}\right|$. However, this procedure does not ensure that $\pi_{r c}$ is a consistent estimator of the break date.

Once the number of factors $r_{a}$ and $r_{b}$ have been consistently estimated by the shrinkage estimator, the classical least squares method in Bai (1997) can be applied to obtain a consistent estimator of the break date. While without knowing the numbers of the pre- and post-break factors, one can not consistently estimate the break date using the least squares criterion, our model selection procedure provides a bridge linking the break-date estimation with observed regressors with break-date estimation in latent factor models.

\section{Monte Carlo Simulations}

In this section, we conduct Monte Carlo simulations to illustrate the accuracy of the proposed model selection procedure, and the mean squared errors (MSEs) of the shrinkage estimators and the PMS estimators in finite samples. Section 7.1 describes the DGPs used in the experiment. Section 7.2 discusses various estimators of the empirical factor models. The simulation results are presented in Section 7.3.

\subsection{Design}

The design of the DGPs roughly follows that in Bates, Plagborg-Møller, Stock, and Watson (2013), with the additional flexibility to accommodate both type-1 and type-2 instabilities 
and the shift of focus from small breaks to large breaks. The DGP takes the form

$$
\begin{array}{cl}
\text { Pre-break: } & X_{i t}=\lambda_{i}^{\prime} F_{t}+e_{i t}, \quad F_{t, \ell}=\rho_{a} F_{t-1, \ell}+\eta_{a} u_{t, \ell}, \\
& t=1, \ldots,\left\lfloor T \pi_{0}\right\rfloor, \quad \ell=1, \ldots, r_{a}, \\
\text { Post-break: } & X_{i t}=\psi_{i}^{\prime} \bar{F}_{t}+e_{i t}, \quad \bar{F}_{t, \ell}=\rho_{b} \bar{F}_{t-1, \ell}+\eta_{b} u_{t, \ell}, \\
& t=\left\lfloor T \pi_{0}\right\rfloor+1, \ldots, T, \quad \ell=1, \ldots, r_{b},
\end{array}
$$

where $i=1, \ldots, N, F_{t}=\left(F_{t, 1}, \ldots, F_{t, r_{a}}\right)^{\prime}$ and $\bar{F}_{t}=\left(\bar{F}_{t, 1}, \ldots, \bar{F}_{t, r_{b}}\right)^{\prime}$. To model the temporal and cross-sectional dependence of the idiosyncratic errors, we consider

$$
e_{i t}=\alpha e_{i t-1}+v_{i t}, v_{t}=\left(v_{1 t}, \ldots, v_{N t}\right)^{\prime} \sim N(0, \Omega)
$$

where the $(i, j)$-th element of $\Omega$ is $\beta^{|i-j|}$. The processes $\left\{u_{t, \ell}: \ell=1, \ldots, r_{b}\right\}$ and $\left\{v_{i t}\right\}$ are mutually independent and are i.i.d. across $t$ with the standard normal distribution. The initial values $F_{0}$ and $e_{0}=\left(e_{10}, \ldots, e_{N 0}\right)^{\prime}$ are drawn from their stationary distribution. When $r_{b}=r_{a}, \bar{F}_{T_{0}}=F_{T_{0}}$. When $r_{b}>r_{a}, \bar{F}_{T_{0}}=\left(F_{T_{0}}^{\prime}, F_{T_{0}}^{*^{\prime}}\right)^{\prime}$, where each element of $F_{T_{0}}^{*}$ is drawn independently from the distribution of $F_{t, \ell}$. The parameters $\left\{N, T, \pi_{0}, r_{a}, r_{b}, \rho_{a}, \rho_{b}, \eta_{a}, \eta_{b}, \alpha, \beta\right\}$ are specified below.

The pre-break factor loadings $\left\{\lambda_{i}: i=1, \ldots, N\right\}$ are independent across $i$ and independent of the factors and the idiosyncratic errors. Let $\lambda_{i} \sim N\left(0, \Sigma_{i}\right)$, where $\Sigma_{i}$ is a diagonal matrix with diagonal elements $\sigma_{i}^{2}(1), \ldots, \sigma_{i}^{2}\left(r_{a}\right)$. These diagonal elements are distinct to ensure that Assumption ID holds, and their sum controls the population regression $R^{2}$ of $X_{i t}$ on the factors. To this end, we set $\sigma_{i}^{2}(\ell)=0.9^{(\ell-1)} \sigma_{i}^{2}(1)$ and $\sum_{\ell=1}^{r_{a}} \sigma_{i}^{2}(\ell)=\sigma^{*}\left(R_{i}^{2}\right)$, where the scalar $\sigma^{*}\left(R_{i}^{2}\right)$ is chosen such that $\mathbb{E}\left[\left(\lambda_{i}^{\prime} F_{t}\right)^{2}\right] / \mathbb{E}\left[X_{i t}^{2}\right]=R_{i}^{2}$ for $t \leq T_{0}$ and $R_{i}^{2}$ is the pre-specified regression $R^{2}$ of the $i$-th series. ${ }^{6}$

We consider two different ways of choosing $R_{i}^{2}$ for $i=1, \ldots, N$. One is the homogeneous case of $R_{i}^{2}=0.5$, which is considered in Bai and $\mathrm{Ng}$ (2002) to assess their information criteria and the benchmark DGP in our simulations. Another is the heterogeneous case in which $R_{i}^{2}$ is calibrated to match the distribution of $R^{2}$ values in the data sets used in the empirical applications. Taking the data set before December 2007, which is the conjectured break date of the recent recession, we regress each time series variable on the principal component estimators of five factors and obtain the empirical distribution of the regression $R^{2}$. We then draw $R_{i}^{2}$ for $i=1, \ldots, N$ independently from this empirical distribution and use the realized $R_{i}^{2}$ to construct the pre-break factor loadings $\lambda_{i}$.

${ }^{6}$ The choice is $\sigma^{*}\left(R_{i}^{2}\right)=\frac{1-\rho_{a}^{2}}{\left(1-\alpha^{2}\right) \eta_{a}^{2}} \frac{R_{i}^{2}}{1-R_{i}^{2}}$. 
Depending on the type of the instabilities, we consider two different ways of constructing the post-break factor loadings $\psi_{i}$. For a type- 1 instability, we set $\psi_{i}=(1-\mathbf{w}) \lambda_{i}+\mathbf{w} \lambda_{i}^{*}$, where $\lambda_{i}^{*}$ and $\lambda_{i}$ are independent and have the same distribution. We vary the scalar $\mathbf{w}$ to control the size of the instability, with $\mathbf{w}=0$ corresponding to the special case of no break in the factor loadings. For a type-2 instability, $\psi_{i}$ is drawn independently of everything else with a distribution that is similar to that of $\lambda_{i}$, except that $r_{a}$ is changed to $r_{b}, \mathbb{E}\left[\left(\psi_{i}^{\prime} \bar{F}_{t}\right)^{2}\right] / \mathbb{E}\left[X_{i t}^{2}\right]=R_{i}^{2}$ for $t>T_{0}$, and the post-December 2007 subsample is used to calibrate $R_{i}^{2}$ in the heterogeneous $R^{2}$ case.

\subsection{Estimators}

We have described the principal component estimators of the factors in the previous sections. The time series variables are normalized to have zero means and unit variances before estimating these factors. ${ }^{7}$ We set the maximum number of factors $k=8$ and standardize the data before getting the principal component estimators. For the data-dependent weight, we set $d=2$.

For experiments with known break dates, model selection is based on the two-step PLS estimator following the algorithm described in Section 5. To investigate the model selection accuracy, we report the probability that the "true" model $\mathcal{M}_{0}=\left(r_{a}, r_{b}, \mathcal{S}_{0}\right)$ is selected, the probabilities of $\widehat{r}_{a}=r_{a}, r_{a}-1$ and $r_{a}+1$, and the probabilities of $\widehat{r}_{b}=r_{b}, r_{b}-1$ and $r_{b}+1$, respectively. In addition, we report MSEs of four different estimators. The first two are the PMS estimator and the PLS estimator described at the end of Section 3. Both of them switch from a full-sample estimation to a subsample estimation only if a break is detected and the number of factors before and after the break are obtained by the shrinkage estimation. In contrast, the third estimator always uses the full sample, and the fourth estimator always uses the post-break subsample. The last two estimators are standard principal component estimators, where the number of factors is selected by the $I C_{p 2}$ information criterion of Bai and Ng (2002). The third estimator coincides with the infeasible benchmark estimator when there is no structural instability, and the fourth estimator coincides with the infeasible benchmark estimator when there is a large change. For the convenience of comparison, the MSE of the first estimator is normalized to be 1 .

\footnotetext{
${ }^{7}$ Without normalization, the idiosyncratic errors of each series have the same variance. When standardizing the variance of all series, those with low regression $R^{2}$ receive more weight. Thus, in the present simulation setup, the procedure performs much better without normalization in the heterogeneous $R^{2}$ case.
} 
For simulations in which the break date is not assumed to be known, the model selection is based on the procedure described in Section 6.3, where $\Pi$ is approximated by a discrete set $\Pi_{d}$. To make them similar to the empirical example investigated in Section 8, we conduct simulations with the true break date at $\pi_{0}=0.8$, and the regression $R^{2}$ is calibrated. The grid size in $\Pi_{d}$ is $\tau=0.01$, a shift by a quarter for a monthly data set of 300 periods, like the data set in our empirical application. We consider $\Pi_{d}=\left\{\pi_{c}-4 \tau, \pi_{c}-3 \tau, \ldots, \pi_{c}, \ldots, \pi_{c}+\right.$ $\left.3 \tau, \pi_{c}+4 \tau\right\}$, which spans a two-year interval and is symmetric around the conjectured break date $\pi_{c}$. We consider both the correct specification case in which $\pi_{c}=\pi_{0}=0.8$ and the misspecification case in which $\pi_{c}=0.78$, which is half a year previous to the real break date in the application. We report model selection probabilities and the MSEs of the four estimators discussed above. To define a post-break subsample for the first two estimators, the revised conjectured break date $\pi_{r c}$ is used because $\pi_{0}$ is unknown. However, to define the post-break subsample for the fourth estimator, we continue to use $\pi_{0}$ because this estimator serves as an infeasible benchmark when there is a large break. For all four estimators, the reported MSEs are based on the subsample from the upper end of $\Pi_{d}$ to the end of the full sample. This subsample ensures the availability of the post-break subsample estimator, no matter where the break date is specified.

\subsection{Monte Carlo Results}

The Monte Carlo results are summarized in four tables. Tables 1 and 2 present results when the break date is known and the regression $R^{2}$ is homogenous across series. The break date is in the middle of the sample in Table $1\left(\pi_{0}=0.5\right)$ and at the end of the sample in Table $2\left(\pi_{0}=0.8\right)$. Table 3 also assumes the break date is known, but the regression $R^{2}$ is calibrated from the data set in the empirical application and thus is heterogeneous across series. Table 4 allows the break date to be unknown, and the regression $R^{2}$ is calibrated. When the regression $R^{2}$ is calibrated, we only conduct simulations with the true break date at the end of the sample, because the potential break occurs around $\pi=0.8$ in the data set of our empirical application. Each table contains three panels, corresponding to no instability, type-1 instability, and type-2 instability, respectively. For a type-1 instability, we consider $\mathbf{w}=0.2,0.5$ and 1 in the DGPs for the scenarios of small, medium, and large changes in the factor loadings, respectively. For a type-2 instability, we consider the changes of the number

of factors from 1 to 2 and 3 to 4 . Various values of $N$ and $T$ are considered. For each DGP, 
Table 1: Known Break Date, Homogeneous $R^{2}, \pi_{0}=0.5$

\begin{tabular}{|c|c|c|c|c|c|c|c|c|c|c|c|c|c|c|c|}
\hline \multicolumn{5}{|c|}{ DGP Configuration } & \multicolumn{4}{|c|}{$\widehat{r}_{a}-r_{a}$} & \multicolumn{3}{|c|}{$\widehat{r}_{b}-r_{b}$} & \multicolumn{4}{|c|}{ MSE } \\
\hline$r_{a}$ & $r_{b}$ & $\mathbf{w}$ & $N$ & $T$ & $\widehat{\mathcal{M}}$ & 0 & -1 & 1 & 0 & -1 & 1 & PMS & PLS & Full & Sub \\
\hline \multicolumn{16}{|c|}{ Panel A. No Change } \\
\hline 3 & 3 & & 100 & 100 & 0.77 & $(0.79$ & 0.21 & $0.00)$ & $(0.96$ & 0.04 & $0.00)$ & 1.00 & 1.07 & 0.83 & 1.35 \\
\hline 3 & 3 & & 150 & 150 & 0.99 & $(0.99$ & 0.01 & $0.00)$ & $(1.00$ & 0.00 & $0.00)$ & 1.00 & 1.01 & 0.99 & 1.59 \\
\hline 3 & 3 & & 200 & 200 & 1.00 & $(1.00$ & 0.00 & $0.00)$ & $(1.00$ & 0.00 & $0.00)$ & 1.00 & 1.01 & 1.00 & 1.60 \\
\hline \multicolumn{16}{|c|}{ Panel B. Type-1 Change } \\
\hline 3 & 3 & 0.2 & 100 & 100 & 0.12 & $(0.88$ & 0.12 & $0.00)$ & $(0.94$ & 0.06 & $0.00)$ & 1.00 & 1.17 & 0.84 & 1.34 \\
\hline 3 & 3 & 0.2 & 150 & 150 & 0.13 & $(1.00$ & 0.00 & $0.00)$ & $(1.00$ & 0.00 & $0.00)$ & 1.00 & 1.30 & 0.95 & 1.30 \\
\hline 3 & 3 & 0.2 & 200 & 200 & 0.13 & (1.00 & 0.00 & $0.00)$ & (1.00 & 0.00 & $0.00)$ & 1.00 & 1.42 & 0.97 & 1.20 \\
\hline 3 & 3 & 0.5 & 100 & 100 & 0.90 & $(0.90$ & 0.10 & $0.00)$ & $(0.94$ & 0.06 & $0.00)$ & 1.00 & 1.11 & 1.42 & 1.15 \\
\hline 3 & 3 & 0.5 & 150 & 150 & 1.00 & (1.00 & 0.00 & $0.00)$ & (1.00 & 0.00 & $0.00)$ & 1.00 & 1.19 & 2.09 & 1.01 \\
\hline 3 & 3 & 0.5 & 200 & 200 & 1.00 & (1.00 & 0.00 & $0.00)$ & (1.00 & 0.00 & $0.00)$ & 1.00 & 1.15 & 2.65 & 1.00 \\
\hline 3 & 3 & 1.0 & 100 & 100 & 0.61 & (0.61 & 0.38 & $0.00)$ & $(0.99$ & 0.01 & $0.00)$ & 1.00 & 0.99 & 1.47 & 1.00 \\
\hline 3 & 3 & 1.0 & 150 & 150 & 0.97 & $(0.97$ & 0.03 & $0.00)$ & (1.00 & 0.00 & $0.00)$ & 1.00 & 0.97 & 1.31 & 1.00 \\
\hline 3 & 3 & 1.0 & 200 & 200 & 1.00 & (1.00 & 0.00 & $0.00)$ & (1.00 & 0.00 & $0.00)$ & 1.00 & 0.98 & 1.30 & 1.00 \\
\hline \multicolumn{16}{|c|}{ Panel C. Type-2 Change } \\
\hline 1 & 2 & & 100 & 100 & 1.00 & $(1.00$ & 0.00 & $0.00)$ & $(1.00$ & 0.00 & $0.00)$ & 1.00 & 0.98 & 1.15 & 1.00 \\
\hline 1 & 2 & & 150 & 150 & 1.00 & (1.00 & 0.00 & $0.00)$ & $(1.00$ & 0.00 & $0.00)$ & 1.00 & 0.98 & 1.13 & 1.00 \\
\hline 1 & 2 & & 200 & 200 & 1.00 & (1.00 & 0.00 & $0.00)$ & $(1.00$ & 0.00 & $0.00)$ & 1.00 & 0.98 & 1.13 & 1.00 \\
\hline 3 & 4 & & 100 & 100 & 0.23 & (0.61 & 0.39 & $0.00)$ & $(0.41$ & 0.58 & $0.00)$ & 1.00 & 1.11 & 1.39 & 0.81 \\
\hline 3 & 4 & & 150 & 150 & 0.90 & $(0.97$ & 0.03 & $0.00)$ & $(0.93$ & 0.07 & $0.00)$ & 1.00 & 1.22 & 1.24 & 0.95 \\
\hline 3 & 4 & & 200 & 200 & 1.00 & (1.00 & 0.00 & $0.00)$ & $(1.00$ & 0.00 & $0.00)$ & 1.00 & 1.13 & 1.23 & 1.00 \\
\hline
\end{tabular}

Notes: Parameters $\alpha=\beta=0.2, \rho_{a}=\rho_{b}=0.5, \eta_{a}=\eta_{b}=1, \zeta=1$. The first five columns are parameters in the DGPs. The next column is the probability that the true model is selected. The next six columns are the probabilities $\widehat{r}_{a}=r_{a}, r_{a}-1, r_{a}+1$, and $\widehat{r}_{b}=r_{b}, r_{b}-1, r_{b}+1$. The last four columns are MSE for the PMS estimator, the PLS estimator, the full-sample estimator, and the post-break subsample estimator.

we report the model selection results and the MSEs of the four estimators introduced above. All results are based on averages over 5,000 simulation repetitions.

Tables S-1 to S-4 in the Appendix contain supplemental results that serve as benchmarks or for robustness checks. Table S-1 is similar to Table 1, but with i.i.d. idiosyncratic errors. Table S-2 is similar to Table 2, but with a different constant $\zeta$ that is associated with larger penalty. Table S-3 is similar to Tables 1 and 2, but the break is in the factor dynamics instead of the loadings, which confirms our early discussion below Assumption ID. Table S-4 is similar to Table 4, but the conjectured break date is equal to the true break date instead 
Table 2: Known Break Date, Homogeneous $R^{2}, \pi_{0}=0.8$

\begin{tabular}{|c|c|c|c|c|c|c|c|c|c|c|c|c|c|c|c|}
\hline \multicolumn{5}{|c|}{ DGP Configuration } & \multicolumn{4}{|c|}{$\widehat{r}_{a}-r_{a}$} & \multicolumn{3}{|c|}{$\widehat{r}_{b}-r_{b}$} & \multicolumn{4}{|c|}{ MSE } \\
\hline$r_{a}$ & $r_{b}$ & $\mathbf{w}$ & $N$ & $T$ & $\widehat{\mathcal{M}}$ & 0 & -1 & 1 & 0 & -1 & 1 & PMS & PLS & Full & Sub \\
\hline \multicolumn{16}{|c|}{ Panel A. No Change } \\
\hline 3 & 3 & & 100 & 200 & 0.99 & $(1.00$ & 0.00 & $0.00)$ & $(1.00$ & 0.00 & $0.00)$ & 1.00 & 1.08 & 0.98 & 2.75 \\
\hline 3 & 3 & & 150 & 300 & 1.00 & $(1.00$ & 0.00 & $0.00)$ & $(1.00$ & 0.00 & $0.00)$ & 1.00 & 1.02 & 1.00 & 2.72 \\
\hline 3 & 3 & & 200 & 400 & .00 & $(1.00$ & 0.00 & $0.00)$ & $(1.00$ & 0.00 & $0.00)$ & 00 & 1.02 & 1.00 & 2.71 \\
\hline \multicolumn{16}{|c|}{ Panel B. Type 1 Change } \\
\hline 3 & 3 & 0.2 & 100 & 200 & 0.12 & $(1.00$ & 0.00 & $0.00)$ & $(1.00$ & 0.00 & $0.00)$ & 1.00 & 1.52 & 0.88 & 1.84 \\
\hline 3 & 3 & 0.2 & 150 & 300 & 0.11 & (1.00 & 0.00 & $0.00)$ & $(1.00$ & 0.00 & $0.00)$ & 1.00 & 1.75 & 0.93 & 1.46 \\
\hline 3 & 3 & 0.2 & 200 & 400 & 0.13 & (1.00 & 0.00 & $0.00)$ & $(1.00$ & 0.00 & $0.00)$ & 1.00 & 2.04 & 0.96 & 1.24 \\
\hline 3 & 3 & 0.5 & 100 & 200 & 1.00 & (1.00 & 0.00 & $0.00)$ & $(1.00$ & 0.00 & $0.00)$ & 1.00 & 1.20 & 1.68 & 1.18 \\
\hline 3 & 3 & 0.5 & 150 & 300 & 1.00 & (1.00 & 0.00 & $0.00)$ & $(1.00$ & 0.00 & $0.00)$ & 1.00 & 1.15 & 2.57 & 1.04 \\
\hline 3 & 3 & 0.5 & 200 & 400 & 1.00 & (1.00 & 0.00 & $0.00)$ & $(1.00$ & 0.00 & $0.00)$ & 1.00 & 1.09 & 3.46 & 1.00 \\
\hline 3 & 3 & 1.0 & 100 & 200 & 1.00 & (1.00 & 0.00 & $0.00)$ & $(1.00$ & 0.00 & $0.00)$ & 1.00 & 0.95 & 2.83 & 1.01 \\
\hline 3 & 3 & 1.0 & 150 & 300 & 1.00 & (1.00 & 0.00 & $0.00)$ & $(1.00$ & 0.00 & $0.00)$ & 1.00 & 0.97 & 2.03 & 1.00 \\
\hline 3 & 3 & 1.0 & 200 & 400 & 1.00 & $(1.00$ & 0.00 & $0.00)$ & $(1.00$ & 0.00 & $0.00)$ & 1.00 & 0.98 & 1.39 & 1.00 \\
\hline \multicolumn{16}{|c|}{ Panel C. Type 2 Change } \\
\hline 1 & 2 & & 100 & 200 & 1.00 & $(1.00$ & 0.00 & $0.00)$ & $(1.00$ & 0.00 & $0.00)$ & 1.00 & 0.96 & 1.68 & 1.00 \\
\hline 1 & 2 & & 150 & 300 & 1.00 & $(1.00$ & 0.00 & $0.00)$ & $(1.00$ & 0.00 & $0.00)$ & 1.00 & 0.97 & 1.18 & 1.00 \\
\hline 1 & 2 & & 200 & 400 & 1.00 & $(1.00$ & 0.00 & $0.00)$ & $(1.00$ & 0.00 & $0.00)$ & 1.00 & 0.98 & 1.14 & 1.00 \\
\hline 3 & 4 & & 100 & 200 & 0.60 & $(1.00$ & 0.00 & $0.00)$ & $(0.60$ & 0.40 & $0.00)$ & 1.00 & 1.05 & 2.83 & 0.97 \\
\hline 3 & 4 & & 150 & 300 & 0.96 & $(1.00$ & 0.00 & $0.00)$ & $(0.96$ & 0.04 & $0.00)$ & 1.00 & 1.08 & 2.92 & 0.99 \\
\hline 3 & 4 & & 200 & 400 & 1.00 & $(1.00$ & 0.00 & $0.00)$ & $(1.00$ & 0.00 & $0.00)$ & 1.00 & 1.03 & 2.24 & 1.00 \\
\hline
\end{tabular}

Notes: Parameters $\alpha=\beta=0.2, \rho_{a}=\rho_{b}=0.5, \eta_{a}=\eta_{b}=1, \zeta=4$.

of being misspecified as in Table 4 .

We now discuss the model selection and estimation results. First, Table 1 shows that our procedure is quite accurate in estimating $r_{a}$ and $r_{b}$ if the break date is in the middle of the sample, even if the number of periods in each subsample is as small as 75 . To detect a type-1 instability, the method works well except when the magnitude of the break is as small as $\mathbf{w}=0.2$. However, the MSE comparison shows that, with a small change of this magnitude, the full-sample estimator yields the smallest MSE, and the PMS estimator benefits from not detecting the break. In the same spirit, when the sample size is as small as 50 in each subsample, our procedure sometimes favors a more parsimonious model by underestimating 
Table 3: Known Break Date, Heterogeneous $R^{2}, \pi_{0}=0.8$

\begin{tabular}{|c|c|c|c|c|c|c|c|c|c|c|c|c|c|c|c|}
\hline \multicolumn{6}{|c|}{ "DGP Configuration } & \multicolumn{3}{|c|}{$\widehat{r}_{a}-r_{a}$} & \multicolumn{3}{|c|}{$\overline{r_{b}-r_{b}}$} & \multicolumn{4}{|c|}{ MSE } \\
\hline$r_{a}$ & $r_{b}$ & $\mathbf{w}$ & $N$ & $T$ & $\widehat{\mathcal{M}}$ & 0 & -1 & 1 & 0 & -1 & 1 & PMS & PLS & Full & Sub \\
\hline \multicolumn{16}{|c|}{ Panel A. No Change } \\
\hline 3 & 3 & & 100 & 200 & 0.97 & $(1.00$ & 0.00 & $0.00)$ & $(1.00$ & 0.00 & $0.00)$ & 1.00 & 1.00 & 0.95 & 3.70 \\
\hline 3 & 3 & & 150 & 300 & 1.00 & $(1.00$ & 0.00 & $0.00)$ & $(1.00$ & 0.00 & $0.00)$ & 1.00 & 1.00 & 1.00 & 3.49 \\
\hline 3 & 3 & & 200 & 400 & 1.00 & $(1.00$ & 0.00 & $0.00)$ & $(1.00$ & 0.00 & $0.00)$ & 1.00 & 1.00 & 1.00 & 3.08 \\
\hline \multicolumn{16}{|c|}{ Panel B. Type-1 Change } \\
\hline 3 & 3 & 0.2 & 100 & 200 & 0.11 & $(1.00$ & 0.00 & $0.00)$ & $(1.00$ & 0.00 & $0.00)$ & 1.00 & 1.02 & 0.78 & 2.66 \\
\hline 3 & 3 & 0.2 & 150 & 300 & 0.01 & (1.00 & 0.00 & $0.00)$ & (1.00 & 0.00 & $0.00)$ & 1.00 & 1.04 & 0.98 & 3.12 \\
\hline 3 & 3 & 0.2 & 200 & 400 & 0.00 & (1.00 & 0.00 & $0.00)$ & (1.00 & 0.00 & $0.00)$ & 1.00 & 1.01 & 1.00 & 2.55 \\
\hline 3 & 3 & 0.5 & 100 & 200 & 0.98 & (1.00 & 0.00 & $0.00)$ & $(1.00$ & 0.00 & $0.00)$ & 1.00 & 0.97 & 0.70 & 0.99 \\
\hline 3 & 3 & 0.5 & 150 & 300 & 1.00 & (1.00 & 0.00 & $0.00)$ & (1.00 & 0.00 & $0.00)$ & 1.00 & 1.39 & 1.13 & 1.36 \\
\hline 3 & 3 & 0.5 & 200 & 400 & 1.00 & (1.00 & 0.00 & $0.00)$ & (1.00 & 0.00 & $0.00)$ & 1.00 & 1.57 & 1.50 & 1.39 \\
\hline 3 & 3 & 1.0 & 100 & 200 & 1.00 & (1.00 & 0.00 & $0.00)$ & $(1.00$ & 0.00 & $0.00)$ & 1.00 & 0.85 & 2.63 & 1.39 \\
\hline 3 & 3 & 1.0 & 150 & 300 & 1.00 & (1.00 & 0.00 & $0.00)$ & (1.00 & 0.00 & $0.00)$ & 1.00 & 0.91 & 4.12 & 1.18 \\
\hline 3 & 3 & 1.0 & 200 & 400 & 1.00 & (1.00 & 0.00 & $0.00)$ & (1.00 & 0.00 & $0.00)$ & 1.00 & 0.93 & 4.74 & 1.03 \\
\hline \multicolumn{16}{|c|}{ Panel C. Type-2 Change } \\
\hline 1 & 2 & & 100 & 200 & 1.00 & $(1.00$ & 0.00 & $0.00)$ & $(1.00$ & 0.00 & $0.00)$ & 1.00 & 0.94 & 2.80 & 1.02 \\
\hline 1 & 2 & & 150 & 300 & 1.00 & $(1.00$ & 0.00 & $0.00)$ & $(1.00$ & 0.00 & $0.00)$ & 1.00 & 0.96 & 1.60 & 1.00 \\
\hline 1 & 2 & & 200 & 400 & 1.00 & $(1.00$ & 0.00 & $0.00)$ & $(1.00$ & 0.00 & $0.00)$ & 1.00 & 0.98 & 1.18 & 1.00 \\
\hline 3 & 4 & & 100 & 200 & 0.54 & $(1.00$ & 0.00 & $0.00)$ & $(0.54$ & 0.46 & $0.00)$ & 1.00 & 0.97 & 3.13 & 1.41 \\
\hline 3 & 4 & & 150 & 300 & 0.93 & $(1.00$ & 0.00 & $0.00)$ & $(0.93$ & 0.07 & $0.00)$ & 1.00 & 1.02 & 3.84 & 1.15 \\
\hline 3 & 4 & & 200 & 400 & 1.00 & (1.00 & 0.00 & $0.00)$ & $(1.00$ & 0.00 & $0.00)$ & 1.00 & 0.99 & 3.53 & 1.01 \\
\hline
\end{tabular}

Notes: Parameters $\alpha=\beta=0.2, \rho_{a}=\rho_{b}=0.5, \eta_{a}=\eta_{b}=1, \zeta=4$.

of the number of factors. Results in Table 1 are for idiosyncratic errors with both temporal and cross-sectional dependence and the default choice of the penalty with $\zeta=1$. If i.i.d. idiosyncratic errors are considered in Table S-1, the procedure works quite well even if each subsample only contains 50 periods and the break is small.

Second, if the break date is at the end of the sample, Table 2 shows a pattern similar to that in Table 1. In particular, when the true model is not selected for a small break or a small sample, the misspecified model typically yields a smaller MSE. Comparing Table 2 with Table S-2, it is clear that $\zeta=4$, which gives a smaller penalty than $\zeta=1$, is preferred 
Table 4: Unknown Break Date, Heterogeneous $R^{2}, \pi_{0}=0.8$

\begin{tabular}{|c|c|c|c|c|c|c|c|c|c|c|c|c|c|c|c|}
\hline \multicolumn{6}{|c|}{ DGP Configuration } & \multicolumn{3}{|c|}{$\overline{\widehat{r}_{a}-r_{a}}$} & \multicolumn{3}{|c|}{$\overline{\widehat{r}_{b}-r_{b}}$} & \multicolumn{4}{|c|}{ MSE } \\
\hline$r_{a}$ & $r_{b}$ & $\mathbf{w}$ & $N$ & $T$ & $\widehat{\mathcal{M}}$ & 0 & -1 & 1 & 0 & -1 & 1 & PMS & PLS & Full & Sub \\
\hline \multicolumn{16}{|c|}{ Panel A. No Change } \\
\hline 3 & 3 & & 100 & 200 & 1.00 & $(1.00$ & 0.00 & $0.00)$ & $(1.00$ & 0.00 & $0.00)$ & 1.00 & 1.01 & 1.00 & 3.90 \\
\hline 3 & 3 & & 150 & 300 & 1.00 & $(1.00$ & 0.00 & $0.00)$ & $(1.00$ & 0.00 & $0.00)$ & 1.00 & 1.00 & 1.00 & 3.49 \\
\hline 3 & 3 & & 200 & 400 & 1.00 & $(1.00$ & 0.00 & $0.00)$ & $(1.00$ & 0.00 & $0.00)$ & 1.00 & 1.00 & 1.00 & 3.08 \\
\hline \multicolumn{16}{|c|}{ Panel B. Type-1 Change } \\
\hline 3 & 3 & 0.2 & 100 & 200 & 0.05 & $(1.00$ & 0.00 & $0.00)$ & $(1.00$ & 0.00 & $0.00)$ & 1.00 & 1.06 & 0.92 & 3.16 \\
\hline 3 & 3 & 0.2 & 150 & 300 & 0.01 & $(1.00$ & 0.00 & $0.00)$ & $(1.00$ & 0.00 & $0.00)$ & 1.00 & 1.03 & 0.99 & 3.16 \\
\hline 3 & 3 & 0.2 & 200 & 400 & 0.00 & $(1.00$ & 0.00 & $0.00)$ & $(1.00$ & 0.00 & $0.00)$ & 1.00 & 1.01 & 1.00 & 2.54 \\
\hline 3 & 3 & 0.5 & 100 & 200 & 0.78 & $(1.00$ & 0.00 & $0.00)$ & $(1.00$ & 0.00 & $0.00)$ & 1.00 & 1.26 & 0.80 & 1.14 \\
\hline 3 & 3 & 0.5 & 150 & 300 & 0.94 & $(1.00$ & 0.00 & $0.00)$ & $(1.00$ & 0.00 & $0.00)$ & 1.00 & 1.92 & 1.19 & 1.43 \\
\hline 3 & 3 & 0.5 & 200 & 400 & 0.99 & $(1.00$ & 0.00 & $0.00)$ & $(1.00$ & 0.00 & $0.00)$ & 1.00 & 2.32 & 1.57 & 1.45 \\
\hline 3 & 3 & 1.0 & 100 & 200 & 1.00 & $(1.00$ & 0.00 & $0.00)$ & $(1.00$ & 0.00 & $0.00)$ & 1.00 & 1.13 & 2.59 & 1.36 \\
\hline 3 & 3 & 1.0 & 150 & 300 & 1.00 & $(1.00$ & 0.00 & $0.00)$ & $(1.00$ & 0.00 & $0.00)$ & 1.00 & 1.12 & 4.06 & 1.17 \\
\hline 3 & 3 & 1.0 & 200 & 400 & 1.00 & $(1.00$ & 0.00 & $0.00)$ & (1.00 & 0.00 & $0.00)$ & 1.00 & 1.07 & 4.69 & 1.02 \\
\hline \multicolumn{16}{|c|}{ Panel C. Type-2 Change } \\
\hline 1 & 2 & & 100 & 200 & 1.00 & $(1.00$ & 0.00 & $0.00)$ & $(1.00$ & 0.00 & $0.00)$ & 1.00 & 0.93 & 2.78 & 1.01 \\
\hline 1 & 2 & & 150 & 300 & 1.00 & $(1.00$ & 0.00 & $0.00)$ & $(1.00$ & 0.00 & $0.00)$ & 1.00 & 0.95 & 1.58 & 1.00 \\
\hline 1 & 2 & & 200 & 400 & 1.00 & $(1.00$ & 0.00 & $0.00)$ & $(1.00$ & 0.00 & $0.00)$ & 1.00 & 0.97 & 1.18 & 1.00 \\
\hline 3 & 4 & & 100 & 200 & 0.39 & $(1.00$ & 0.00 & $0.00)$ & $(0.54$ & 0.46 & $0.00)$ & 1.00 & 0.98 & 3.00 & 1.36 \\
\hline 3 & 4 & & 150 & 300 & 0.86 & $(1.00$ & 0.00 & $0.00)$ & $(0.93$ & 0.07 & $0.00)$ & 1.00 & 1.04 & 3.71 & 1.11 \\
\hline 3 & 4 & & 200 & 400 & 1.00 & $(1.00$ & 0.00 & $0.00)$ & $(1.00$ & 0.00 & $0.00)$ & 1.00 & 1.00 & 3.50 & 1.00 \\
\hline
\end{tabular}

Notes: Parameters $\alpha=\beta=0.2, \rho_{a}=\rho_{b}=0.5, \eta_{a}=\eta_{b}=1, \zeta=4$. The conjecture break date $\pi_{c}$ is misspecified, $\pi_{c}=0.78$.

if the break date is moved from the middle of the sample to the end of the sample. This is particularly important to detect a type-2 instability, because the new factors are only in the post-break subsample, which has a small sample size. If there is no structural break or if there is a type- 1 instability, model selection results are more robust to the choice of $\zeta$.

Third, Table 3 and Table 4 show that heterogeneous regression $R^{2}$ and unknown break date make the model selection procedure less accurate than that in Table 2, but it still works quite well in general. In all cases, $r_{a}$ can be accurately estimated, even if the postbreak subsample is as small as 40 . In a factor model with type- 2 instability, $r_{b}$ might be 
underestimated if the post-break subsample is 40, but this underestimation issue is minor if the size of the post-break subsample is larger than 80. As in other cases, type-1 instability can be detected except when $\mathbf{w}=0.2$. Table 4 and Table $\mathrm{S}-4$ suggest that the results are similar with different specifications of the conjectured break date.

Finally, the PMS estimator (the first column) switches between the full-sample estimator (the third column) and the post-break subsample estimator (the fourth column) and when evaluated by its MSE, the PMS estimator may have better finite sample performances than the other two estimators in some scenarios. For example, in Panel B of Table 1 with $\mathbf{w}=0.5$, $N=100$, and $T=100$, the MSEs of the full-sample estimator and the post-break subsample estimator, respectively, are $42 \%$ and $15 \%$ larger than that of the PMS estimator. The PLS estimator (second column) may have a smaller MSE than the PMS estimator. This could happen for a type-1 instability with a large $\mathbf{w}$ or a type- 2 instability where the factor loadings of the new factors are large. On the other hand, when the shrinkage causes much more bias than desired, the PLS estimator can be worse than the PMS estimator.

\section{Structural Changes During the Great Recession}

Unlike in other post-war recessions, the disruption of borrowing and lending played an important role in the 2007-2009 recession. Narratives emphasize a collapse of the U.S. housing market; massive devaluations of mortgage-backed securities that spilled over to other asset markets and ultimately led to a large-scale disruption of financial intermediation; a drop in real activity caused by the crisis in the financial sector; and an extended period of zero nominal interest rates in combination with unconventional monetary policy interventions. We use the shrinkage methods developed in the preceding sections to investigate the stability of factor loadings and the emergence of new factors. Section 8.1 describes the data set. Estimates of the number of pre-2007 and post-2007 factors are presented in Section 8.2. Finally, we make some identification assumptions and provide an interpretation of the estimated factors in Section 8.3.

\subsection{Data Set}

The data set used for the empirical analysis is based on Stock and Watson (2012), who compiled a set of 200 macroeconomic and financial indicators. These 200 series contain both 
Table 5: Categories of Time Series

\begin{tabular}{llr}
\hline \hline Symbol & Description & Series \\
\hline NIPA & National Income and Product Accounts & 5 \\
IP & Industrial Production & 9 \\
Emp & Employment and Unemployment & 30 \\
HSS & Housing Starts & 6 \\
Ord & Orders, Inventories, and Sales & 7 \\
Pri & Productivity & 22 \\
IntL & Interest Rates (Level) & 2 \\
IntS & Interest Rates (Spread) & 7 \\
Mon & Money and Credit & 5 \\
StPr & Stock Prices and Wealth & 3 \\
ExR & Exchange Rates & 5 \\
Others & Consumer Expectation & 1 \\
\hline
\end{tabular}

high-level aggregates and disaggregated components. To avoid double counting, Stock and Watson retained 132 of the 200 series, and we refer to the resulting data set as SW132. Using SW132 as starting point, our data set is constructed as follows: (i) We extend the series in the SW132 data set to 2012:M12, using May 2013 data vintages. (ii) We replace the quarterly series in SW132 by their monthly counterparts, if available. This is possible for consumption of nondurables, services, and durables; for nonresidential investment; and for 16 price series. We remove the remaining quarterly series for which no monthly observations are available. (iii) We add two GDP components that are available at monthly frequency: change in private inventory and wage and salary disbursements. (iv) Following Stock and Watson (2012), we remove local means from all series using a biweight kernel with a bandwidth of 100 months. The local means are approximately the same as the ones obtained by a centered moving average of \pm 70 months. After making these modifications, our data set consists of $N=102$ series of monthly macroeconomic and financial indicators, which are grouped into the 12 categories listed in Table 5. The sample begins after the Great Moderation and ranges from 1985:M1 to 2013:M1 $(T=337)$. 
Table 6: Model Selection, $T_{c}$ is 2007:12

\begin{tabular}{|c|c|c|c|c|c|}
\hline \multicolumn{2}{|c|}{ Penalty } & \multicolumn{4}{|c|}{ Estimates } \\
\hline Scaling $\zeta$ & Interval & Break $T_{r c}$ & $\hat{r}_{a}$ & $\left(\hat{r}_{b}-\hat{r}_{a}\right)$ & $\left\|\widehat{\Gamma}_{l}\right\|^{2}$ \\
\hline \multicolumn{6}{|c|}{ Panel A. Known Break Date } \\
\hline 1 & $\mathrm{~N} / \mathrm{A}$ & 2007:M12 & 1 & 1 & 3.66 \\
\hline 4 & $\mathrm{~N} / \mathrm{A}$ & 2007:M12 & 4 & 1 & 5.02 \\
\hline 6 & $\mathrm{~N} / \mathrm{A}$ & 2007:M12 & 6 & 0 & 5.52 \\
\hline \multicolumn{6}{|c|}{ Panel B. Unknown Break Date } \\
\hline 1 & $T_{c} \pm 6$ & 2007:M12 & 1 & 1 & 12.98 \\
\hline 1 & $T_{c} \pm 12$ & 2007:M12 & 1 & 1 & 15.38 \\
\hline 4 & $T_{c} \pm 6$ & 2007:M12 & 3 & 0 & 19.20 \\
\hline 4 & $T_{c} \pm 12$ & 2007:M3 & 3 & 0 & 24.31 \\
\hline 6 & $T_{c} \pm 6$ & 2007:M6 & 5 & 1 & 19.86 \\
\hline 6 & $T_{c} \pm 12$ & 2007:M5 & 5 & 1 & 25.19 \\
\hline
\end{tabular}

Notes: The PLS estimator uses the averaging penalty functions $P_{1}^{*}(\Lambda)$ and $P_{2}^{*}(\Lambda)$ defined in (6.10) where the average is taken over the interval specified in the second column of this table.

\subsection{The Number of Factors Before and After 2007:M12}

The empirical analysis is based on the two-step estimation procedure described in Section 5.2. The starting point is a conjectured break date $T_{c}=2007$ :M12, which is the beginning of the Great Recession, according to the business cycle dating of the National Bureau of Economic Research (NBER). We use the extensions described in Section 6 to account for the fact that the "true" break date is unknown. Throughout the empirical analysis, we fix the number of potential factors to $k=8$, and we set the constant that controls the rate of decay (as a function of the sample size) of the penalty term to $d=2$, as we did for the Monte Carlo analysis in Section 7. To document the sensitivity of the empirical results to the magnitude of the penalty terms $P_{1}(\Lambda)$ and $P_{2}(\Lambda)$ (or $P_{1}^{*}(\Lambda)$ and $P_{2}^{*}(\Lambda)$ for the case of unknown break point), we vary the scaling constant $\zeta$ that appears in the definition of the penalty weights $\alpha_{N T}$ and $\beta_{N T}$ in (5.6).

The model selection results are summarized in Table 6 for different choices of the penalty scaling factor $\zeta$. We distinguish the case of treating the break date as known (Panel A) from the case of an unknown break date (Panel B). Following the procedure described in 
Section 6.3, we consider break dates $T_{a}$ over the range $T_{c} \pm \bar{\tau}$, where $\bar{\tau}$ is either six or 12 months, and generate estimates of $\hat{r}_{a}\left(T_{a} / T\right)$ and $\hat{r}_{b}\left(T_{b} / T\right)$, keeping the overall sample size $T=T_{a}+T_{b}$ fixed. If $\hat{r}_{a}\left(T_{a} / T\right)+\hat{r}_{b}\left(T_{b} / T\right)$ achieves its minimum at $T_{c}$ then we do not revise the conjectured break date. If the minimum is achieved elsewhere in the interval $T_{c} \pm \bar{\tau}$, we use the date closest to the conjectured break date at which the minimum is achieved. For $\zeta=1$, the conjectured break date is not revised, whereas for larger values of $\zeta$, in particular $\zeta=6$, the break date is shifted several months backward in time to the first half of 2007 .

We subsequently focus on the case of an unknown break date. The estimates of the number of factors is robust to the choice of $\bar{\tau} \in\{6,12\}$. The overall number of factors is increasing in the scaling factor $\zeta$, because the larger $\zeta$ the smaller the penalty for nonzero coefficients. By choosing different values for $\zeta$, we are essentially setting different thresholds for the increase in goodness-of-fit that an additional factor must generate to justify its inclusion. For the pre-2007 sample, the number of factors ranges from $\hat{r}_{a}=1$ for $\zeta=1$ to $\hat{r}_{a}=5$ for $\zeta=6$. For comparison, we also estimated the number of pre-break factors using the Bai and $\mathrm{Ng}(2002)$ criteria: $I C_{1}$ and $I C_{2}$ deliver the estimate $\tilde{r}_{a}=1$, whereas $I C_{3}$ generates either $\tilde{r}_{a}=6$ (sample ending in 2007:M5 or 2007:M6) or $\tilde{r}_{a}=7$ (sample ending in 2007:M3 or 2007:M12).

While the estimation of the overall number of factors is fairly sensitive to the choice of $\zeta$, the estimate of the change in the number of factors, $\hat{r}_{b}-\hat{r}_{a}$ is quite stable. For $\zeta=1$ and $\zeta=6$, we detect a type-2 instability and find that the number of factors post-2007 has increased by one. For $\zeta=4$, our procedure detects a type-1 instability, meaning that the loadings change but the estimated number of factors stays constant.

Using the estimates for $\zeta=6$ and $T_{c} \pm 12$, we now decompose the effect of the structural change into the effect of the change in loadings on the old factors and the effect of the new factor. The decomposition is based on (2.14). As a baseline, we compute $R^{2}$ values for each individual series based on the variation explained by $F_{b, 1}^{R \Omega} \Lambda^{R^{\prime}}+F_{b, 1}^{R \Omega}\left(\Psi_{1}^{R \Omega}-\Lambda^{R}\right)^{\prime}($ new loadings only). We compare the baseline $R^{2}$ s to $R^{2}$ s associated with $F_{b, 1}^{R \Omega} \Lambda^{R^{\prime}}$ (old loadings) and $R^{2}$ s associated with all three terms in (2.14) (i.e., new loadings and factor). The results are plotted in Figure 1. Bars below the zero baseline indicate the $R^{2}$ loss due to ignoring the change in loadings. Bars above the zero line indicate the $R^{2}$ gain from also accounting for the effect of the new factor. Each set of bars corresponds to an individual series, and the vertical lines delimit the time series categories listed in Table 5. 
Figure 1: $R^{2}$ Gains from New Loadings And Factor: $\left(\hat{r}_{1}, \hat{r}_{2}\right)=(5,6)$

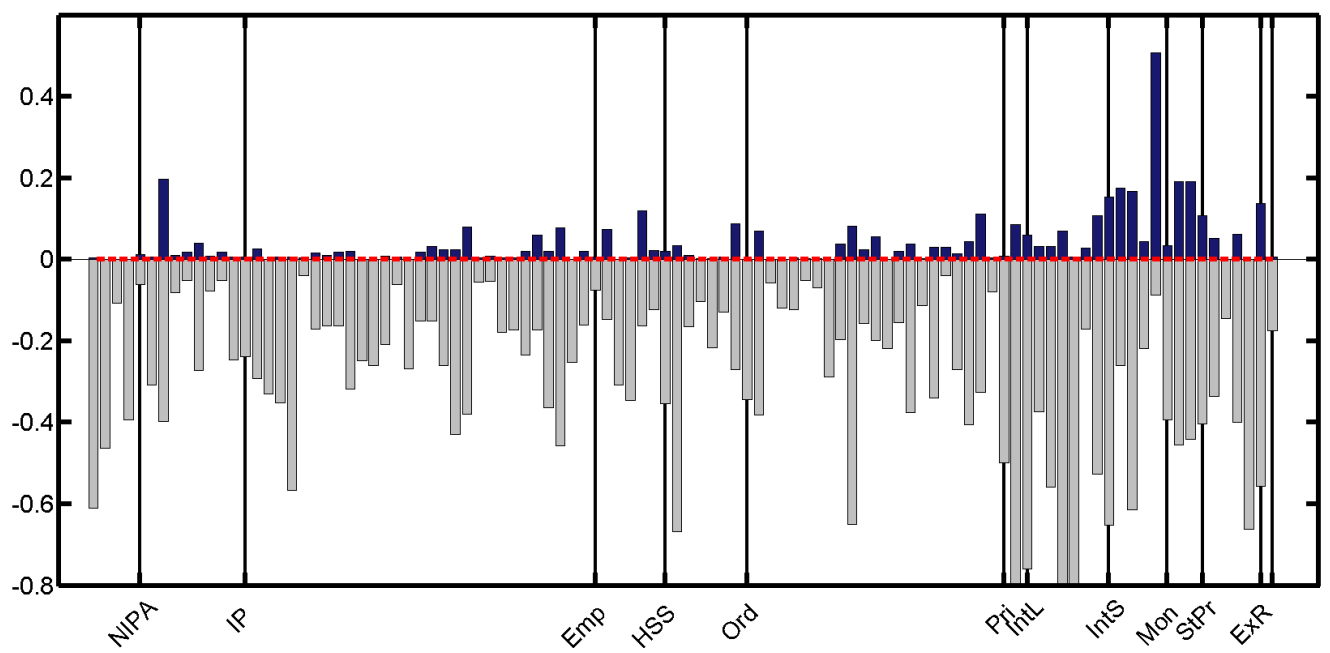

Notes: Base line case new loadings only. Dark bars (above zero) indicate $R^{2}$ gain due to new factor relative to new loadings only. Light grey bars (below zero) indicate $R^{2}$ losses from using the old loading matrices. The procedure with $\zeta=6$ and interval $2007: M 12 \pm 12$ selects the break date 2007:M5.

Two observations stand out. First, our estimates attribute most of the structural change to a change in the loadings of the existing factors, in the sense that the contribution of $F_{b, 1}^{R \Omega}\left(\Psi_{1}^{R \Omega}-\Lambda^{R}\right)^{\prime}$ to the overall $R^{2}$ exceeds the contribution of $F_{b, 2}^{R \Omega} \Psi_{2}^{R \Omega^{\prime}}$. This is consistent with the fact that for $\zeta=4$, our estimate of the change in the number of factors is equal to zero. The effect of the loadings change dominates. Second, the new factor mainly affects financial variables, namely those series in the two interest rate categories (IntL, IntS), the money and credit group (Mon), and the stock price and wealth group (StPr). While there are some spillovers to the real side (i.e., industrial production (IP), employment (EMP), and housing starts (HSS) variables), these spillovers are relatively small and affect only a fraction of the series.

Figure 2 depicts the fitted time path of four series: the spread between commercial paper and Treasury bills, housing starts in the southern Census district, capital utilization, and employment in durable goods manufacturing. We overlay the actual sample paths with three (in-sample) predicted paths, which, as before, we refer to as old loadings, new loadings only, new loadings and factor. The spread starts to rise toward the end of 2007. This rise is not captured by the path predicted under the pre-break loadings, which stays fairly constant 
Figure 2: In-SAMPle PREDiCTION: Individual SERIES

Spread (CP-Tbill)

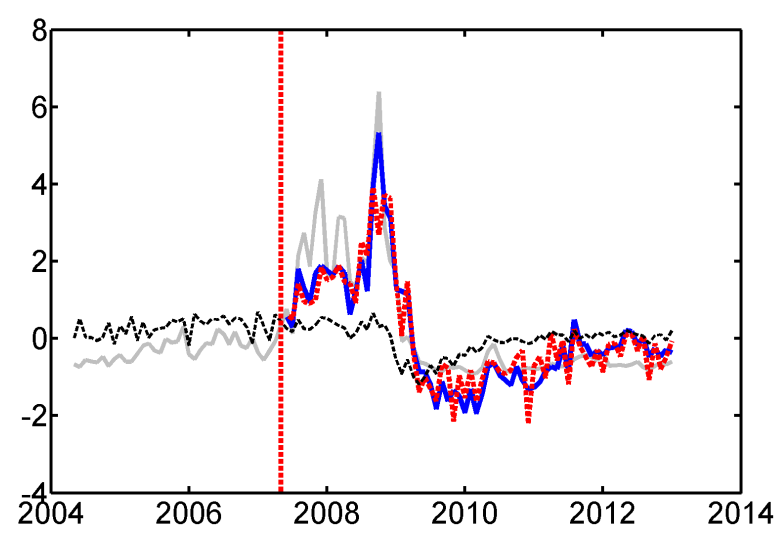

Capital Utilization (Total)

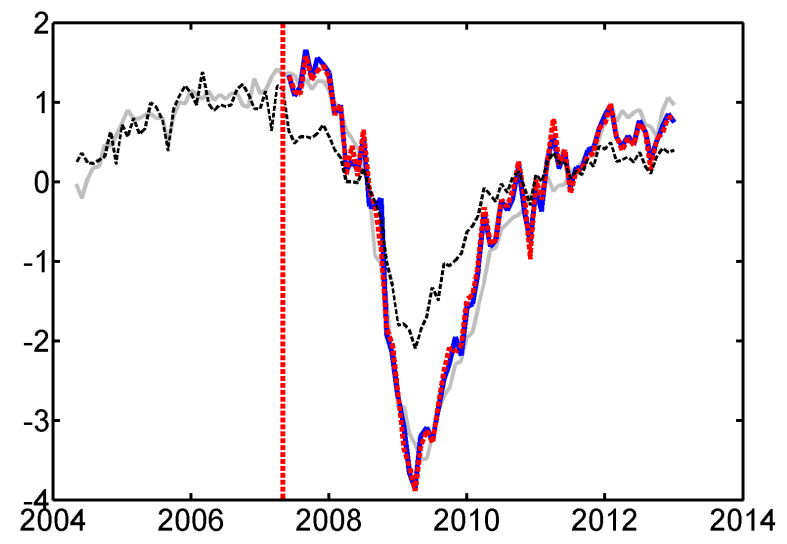

Housing Starts (South)

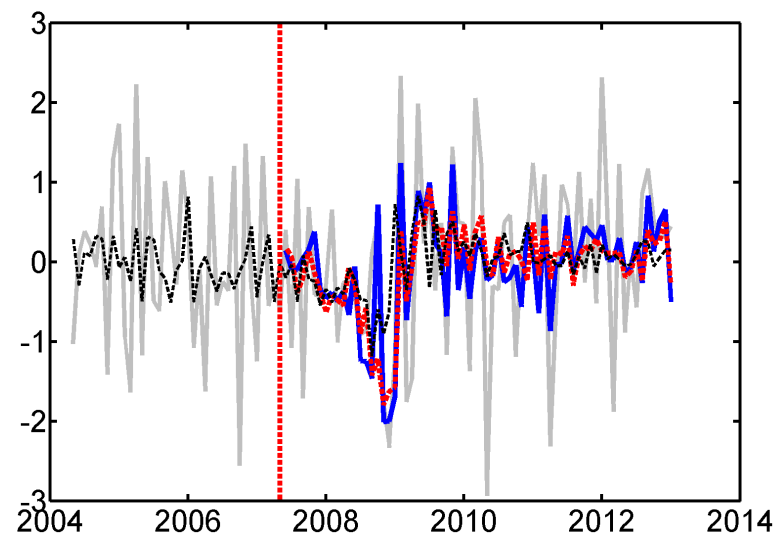

Employment (Durable Goods)

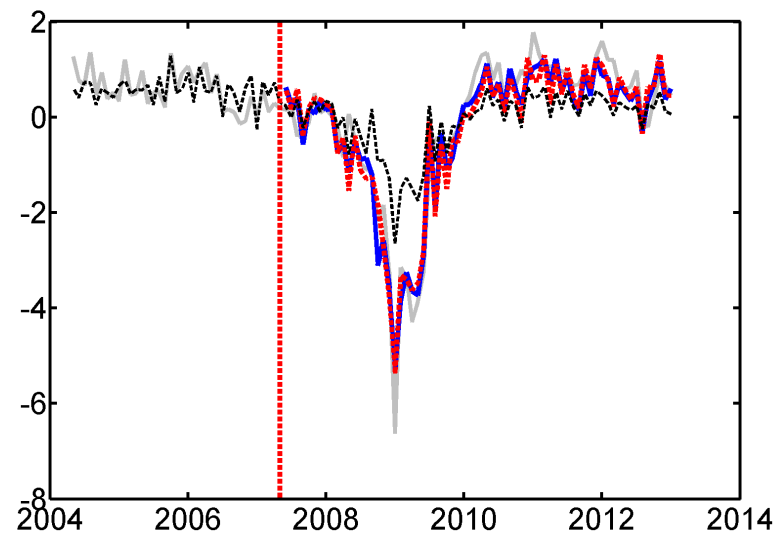

Notes: Gray line: actual; black dashed: old loadings; thick dashed red: new loadings only; thick blue: new loadings and factor. Break date is 2007:M5 and $\left(r_{1}, r_{2}\right)=(5,6)$. Our procedure with $\zeta=6$ and interval $2007 M 12 \pm 12$ selects $\left(r_{1}, r_{2}\right.$, dates $)=(5,6,2007: \mathrm{M} 5)$.

throughout 2008. As suggested by Figure 1, the discrepancy between the old loadings and the new loadings only paths is substantial during the Great Recession period. Once the loadings are allowed to change, the predicted spread rises drastically throughout 2008, and even more so once the new factor is accounted for. Capital utilization and employment drop drastically in the second half of 2008 and only start recovering in 2010. The old loadings path is unable to capture the large drop in real activity. With the new loadings only, on the other hand, the model is able to track both capital utilization and employment quite well 
during and after the recession, and the additional factor has not altered the predicted paths of these series. However, there are real series that show a noticeable effect of the new factor, one of them being the housing starts series in the top right panel of Figure 2.

At first glance, the results in Figure 2 look very different from those presented in Figure 2 of Stock and Watson (2012). Part of the discrepancy is due to the different normalization schemes. We are normalizing the variance of the factors to one, whereas Stock and Watson (2012) normalized the length of the loading vectors to one (i.e., $\Lambda^{\prime} \Lambda / N=I_{r}$ ). To be able to explain the macroeconomic dynamics during and after the Great Recession with factors that have unit variance, a big change in the loadings is required. This is evident from our Figures 1

and 2. If we normalize the length of the loadings before and after the break to one, then the increase in the volatility after 2007 is absorbed in an increase in the factor volatility. The ratio of pre- to post-break factor variance under this alternative normalization ranges from 1.26 to 1.93 for the five factors that were active prior to the break. Stock and Watson (2012) interpret this phenomenon as an unchanged response to "old" factors combined with large innovations to the "old" factors in the post-2007 sample. In the absence of the emergence of a new factor, we would interpret this phenomenon as a type- 1 instability of the factor model.

To summarize, our model selection procedure provides strong evidence that the loadings in the normalized factor model changed drastically, generally implying a stronger comovement of the series after 2007. There is also some evidence of the emergence of a new factor, which to a large extent seems to capture important co-movements among financial series but also spills over into the real activity variables. While the estimate of the total number of factors is sensitive to the scaling of the penalty terms in the objective function of the PLS estimator, the estimate of the number of new factors, which is either one or zero, is much more robust to the tuning of the penalty terms.

\subsection{Interpreting the Old and New Factors}

Previously, our analysis focused on determining the number of factors pre- and post 2007:M12 and the type of the structural instability. Moreover, for the detected type-2 structural changes we decomposed the overall break effect into the contribution of breaks in the loadings and the emergence of a new factor. Due to the normalization imposed on the DGP, the estimated pre- and post-break factors have identity covariance matrices and no specific 
economic interpretation. We will now use a nonsingular $r_{a} \times r_{a}$ matrix $B\left(r_{b} \times r_{b}\right.$ matrix $B$ ) to transform the estimated pre-break factors $\tilde{F}_{a}$ (post-break factors $\tilde{F}_{b}$ ) into factors $\widetilde{F}_{a}^{\dagger}$ $\left(\widetilde{F}_{b}^{\dagger}\right)$ that resemble the first principal components of the variable groups listed in Table 5 and therefore can be interpreted as NIPA, IP, EMP, etc. ${ }^{8}$

To simplify the notation, we drop the "hats" and "tildes" from matrices associated with sample estimates. Without loss of generality, we assume that the columns of $X_{a}$ are arranged according to the $J=11$ categories listed in Table 5 , such that we can partition $X_{a}=$ $\left[X_{a, 1}, \ldots, X_{a, J}\right]$, where $X_{a, j}$ contains the series associated with the $j$ 'th category of variables, e.g., $X_{a, 1}$ comprises the five NIPA series. For each $X_{a, j}$ we calculate the first principal component, denoted by the $T \times 1$ vector $x_{a, j}$, which can be interpreted as a group-specific factor. We then project each group-specific factor on the space spanned by $F_{a}$ :

$$
\xi_{a, j}=F_{a} B_{j}+\text { resid }, \quad j=1, \ldots, J
$$

and refer to the $T \times 1$ vector of predicted values $F_{a} \hat{B}_{j}$ as the NIPA factor for $j=1$, the IP factor for $j=2$, and so forth. For each regression $j$ in (8.1), we compute the associated $R^{2}$ and then select the $r_{a}$ categories that deliver the highest $R^{2}$ values. The associated coefficient estimates are $\hat{B}_{(1)}, \ldots, \hat{B}_{\left(r_{a}\right)}$, where each $\hat{B}_{(j)}$ is a $r_{a} \times 1$ vector. In turn, we define

$$
B=\left(\hat{B}_{(1)}, \ldots, \hat{B}_{\left(r_{a}\right)}\right), \quad F_{a}^{\dagger}=F_{a} B, \quad \text { and } \quad \Lambda^{\dagger^{\prime}}=B^{-1} \Lambda^{\prime}
$$

The transformed factors are labeled according to the associated $\xi_{a,(j)}$ vectors.

For the post-break period, we have a $T \times r_{b}$ matrix of estimated factors $F_{b}$, which we will now transform into a set of economically interpretable factors $F_{b}^{\dagger}=F_{b} C$ using a nonsingular $r_{b} \times r_{b}$ matrix $C$. First, we extend the transformed pre-break factors $F_{a}^{\dagger}$ into the post-break period. Note that $F_{a}^{\dagger}$ consists of linear combinations of $X_{a}$, say, $F_{a}^{\dagger}=X_{a} \Upsilon_{a}^{\dagger}$. Thus, we can define $F_{b \mid a}^{\dagger}=X_{b} \Upsilon_{a}^{\dagger}$. The extended factors $F_{b \mid a}^{\dagger}$ need not fall into the space spanned by the post-break factors $F_{b}$. Thus, we project the extended factors on the space spanned by $F_{b}$ using the following regression:

$$
F_{b \mid a}^{\dagger}=F_{b} C_{0}+\text { resid }
$$

Here the matrix $C_{0}$ is of dimension $r_{b} \times r_{a}$. If $r_{b}>r_{a}$, we proceed by computing post-break group-specific factors $\xi_{b, j}$ based on the first principal components. Focusing on the variable

\footnotetext{
${ }^{8}$ An alternative method of constructing interpretable factors is developed in Dobrev and Schaumburg
} (2013). 
Table 7: Rotated Factors, Unknown Break Date, $T_{c}$ Is 2007:12

\begin{tabular}{cccccccc}
\hline \hline \multicolumn{2}{c}{ Penalty } & \multicolumn{9}{c}{ Pre-Break Factors } & & New Post-Break \\
Scaling $\zeta$ & Interval & $F_{1}$ & $F_{2}$ & $F_{3}$ & $F_{4}$ & $F_{5}$ & $F_{1}^{*}$ \\
\hline 1 & $T_{c} \pm 12$ & Emp & & & & & IntS \\
6 & $T_{c} \pm 12$ & Emp & IP & IntS & ExR & Pri & Mon \\
\hline
\end{tabular}

groups $j$ that were not used in the construction of the rotated pre-break factors, we estimate

$$
\xi_{b, j}=F_{b} C_{j}+\text { resid }
$$

and compute the $R^{2}$ associated with this regression. Let $\left(r_{a}+1\right), \ldots,\left(r_{b}\right)$ denote the indices that generate the highest $R^{2}$ values. We define the post-break transformation and the associated transformed factors and loadings as

$$
C=\left(\hat{C}_{0}, \hat{C}_{\left(r_{a}+1\right)}, \ldots, \hat{C}_{\left(r_{b}\right)}\right), \quad F_{b}^{\dagger}=F_{b} C, \quad \text { and } \quad \Psi^{\dagger^{\prime}}=C^{-1} \Psi^{\prime}
$$

Table 7 summarizes the labels for the transformed pre-break factors and the additional post-break factors for the two choices of $\zeta$ under which a new factor was detected. Since the results were identical for $T_{c} \pm 6$ and $T_{c} \pm 12$, we only report the latter. For $\zeta=1$, we identify the pre-break factor as an employment factor. By assumption, the employment factor continues to be active from 2008:M1 onward but a second factor, namely an interest rate spread factor, emerges. This is consistent with the narrative of a financial crisis in which drastic increases in spreads coincide with substantial drops in real activity.

For $\zeta=6$, our identification scheme labels the five pre-break factors as employment, industrial production, interest rates, exchange rates, and prices. We verified that the transformed factors track the group-specific factors well. For the categories employment, industrial production, and money and credit, the correlation with the group-specific factors is above 0.9. The new post-break factor is money and credit, which is also broadly consistent with the narrative of the Great Recession. Overall, our analysis suggests that one can construct a plausible identification scheme for the factors under which the new factor that emerged during the Great Recession can be broadly interpreted as a financial factor. This conclusion is consistent with the findings in Figure 1, in which the financial variables are associated with the largest $R^{2}$ gains from the new factor. 


\section{Conclusion}

We develop a shrinkage estimation procedure for high-dimensional factor models that generates consistent estimates of the number of pre- and post-break factors. In situations in which the number of factors is constant throughout the sample, the procedure can consistently detect changes in the matrix of factor loadings. Our model selection procedure remains consistent even if the break date is unknown; however, it does not generate a consistent estimate of the break date itself. Nevertheless, once the number of pre- and post-break factors is known, conventional methods can be used to estimate the break date consistently. Our Monte Carlo analysis shows that the procedure has good finite sample properties. In an application to U.S. data, we show that the procedure detects an increase in the number of factors for a large macroeconomic and financial data set at the onset of the Great Recession. After imposing some identification conditions, we show that the new factor can be interpreted as a financial factor, which is consistent with the narratives of the 2007-2009 recession.

\section{References}

Ahn, S. C., And A. R. Horenstein (2013): "Eigenvalue Ratio Test for the Number of Factors," Econometrica, 81(3), 1203-1227.

Alessi, L., M. Barigozzi, And M. Capasso (2010): "Improved Penalization for Determining the Number of Factors in Approximate Factor Models," Statistics 85 Probability Letters, 80(23-24), 1806-1813.

Amengual, D., And M. W. Watson (2007): "Consistent Estimation of the Number of Dynamic Factors in a Large N and T Panel," Journal of Business 6 Economic Statistics, 25(1), 91-96.

BAI, J. (1997): "Estimation of a Change Point in Multiple Regression Models," Review of Economics and Statistics, 79(4), 551-563.

_ (2003): "Inferential Theory for Factor Models of Large Dimensions," Econometrica, $71(1), 135-171$. 
BAI, J., AND Y. LiaO (2012): "Efficient Estimation of Approximate Factor Models via Regularized Maximum Likelihood," Manuscript, Columbia University and University of Maryland.

BAI, J., AND S. NG (2002): "Determining the Number of Factors in Approximate Factor Models," Econometrica, 70(1), 191-221.

(2007): "Determining the Number of Primitive Shocks in Factor Models," Journal of Business $\&$ Economic Statistics, 25, 52-60.

(2013): "Principal Components Estimation and Identification of Static Factors," Journal of Econometrics, 176(1), 18-29.

Bates, B. J., M. Plagborg-Møller, J. H. Stock, and M. W. Watson (2013): "Consistent Factor Estimation in Dynamic Factor Models with Structural Instability," Journal of Econometrics, 177(2), 289-304.

Breitung, J., And S. Eickmeier (2011): "Testing for Structural Breaks in Dynamic Factor Models," Journal of Econometrics, 163(1), 71-84.

Breitung, J., And U. Pigorsch (2013): "A Canonical Correlation Approach for Selecting the Number of Dynamic Factors," Oxford Bulletin of Economics and Statistics, 75(1), 2336.

Bühlmann, P., And S. VAn DE GeER (2011): Statistics for High-Dimensional Data: Methods, Theory and Applications. New York: Springer.

Caner, M., And X. Han (2012): "Selecting the Correct Number of Factors in Approximate Factor Models: The Large Panel Case with Group Bridge Estimators," Manuscript, North Carolina State University.

Chen, L., J. J. Dolado, and J. Gonzalo (2011): "Detecting Big Structural Breaks in Large Factor Models," Manuscript, Universidad Carlos III de Madrid.

Chor, I. (2013): "Model Selection for Factor Analysis: Some New Criteria and Performance Comparisons," Working Paper, Sogang University Research Institute for Market Economy, $1209(1209)$.

Cliff, N. (1966): "Orthogonal Rotation to Congruence," Psychometrika, 31(1), 33-42. 
Corradi, V., and N. Swanson (2013): "Testing for Structural Stability of Factor Augmented Forecasting Models," Journal of Econometrics, forthcoming.

Dobrev, D., And E. Schaumburg (2013): "Robust Forecasting by Regularization," Manuscript, Board of Governors of the Federal Reserve System and Federal Reserve Bank of New York.

Forni, M., M. Hallin, M. Lippi, and L. Reichlin (2000): "The Generalized Dynamic Factor Model: Identification and Estimation," Review of Economics and Statistics, 82(4), 540-554.

Hallin, M., And R. LikA (2007): "Determining the Number of Factors in the General Dynamic Factor Model," Journal of the American Statistical Association, 102(478), 603617.

Han, X., And A. Inoue (2011): "Tests for Parameter Instability in Dynamic Factor Models," Manuscript, North Carolina State University.

Kapetanios, G. (2010): "A Testing Procedure for Determining the Number of Factors in Approximate Factor Models With Large Datasets," Journal of Business Ef Economic Statistics, 28(3), 397-409.

Lee, S., M. H. Seo, and Y. Shin (2012): "The Lasso for High-Dimensional Regression with a Possible Change-Point," ArXiv e-prints http://arxiv.org/abs/12094875.

Lu, X., ANd L. Su (2013): "Shrinkage Estimation of Dynamic Panel Data Models with Interactive Fixed Effects," Manuscript, Hong Kong University of Science \& Technology and Singapore Management University.

Onatski, A. (2009): "Testing Hypotheses About the Number of Factors in Large Factor Models," Econometrica, 77(5), 1447-1479.

— (2010): "Determining the Number of Factors from Empirical Distribution of Eigenvalues," Review of Economics and Statistics, 92(4), 1004-1016.

(2012): "Asymptotics of the Principal Components Estimator of Large Factor Models with Weakly Influential Factors," Journal of Econometrics, 168(2), 244-258. 
QiAn, J., AND L. Su (2013): "Shrinkage Estimation of Regression Models with Multiple Structural Change," Manuscript, Shanghai Jiao Tong University and Singapore Management University.

Schönemann, P. (1966): "A Generalized Solution of the Orthogonal Procrustes Problem," Psychometrika, 31(1), 1-10.

Stock, J. H., And W. M. Watson (2002): "Forecasting Using Principal Components From a Large Number of Predictors," Journal of the American Statistical Association, $97(460), 1167-1179$.

(2009): "Forecasting in Dynamic Factor Models Subject to Structural Instability," in The Methodology and Practice of Econometrics: Festschrift in Honor of D.F. Hendry, ed. by N. Shephard, and J. Castle, pp. 1-57. Oxford University Press.

- (2012): "Disentangling the Channels of the 2007-09 Recession," Brookings Papers on Economic Activity, pp. 81-156.

TibshiRAni, R. (1994): "Regression Shrinkage and Selection Via the Lasso," Journal of the Royal Statistical Society, Series B: Statistical Methodology, 58(1), 267-288.

YUAN, M., AND Y. LIN (2006): "Model selection and estimation in regression with grouped variables," Journal of the Royal Statistical Society, Series B: Statistical Methodology, 68(1), 49-67.

Zou, H. (2006): "The Adaptive Lasso and Its Oracle Properties," Journal of the American Statistical Association, 101(476), 1418-1429. 